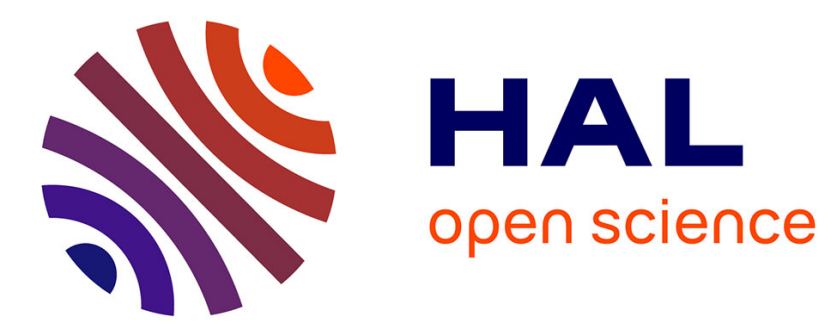

\title{
ENVELOPE THEOREMS FOR STATIC OPTIMIZATION AND CALCULUS OF VARIATIONS
}

Joël Blot, Hasan Yilmaz

\section{To cite this version:}

Joël Blot, Hasan Yilmaz. ENVELOPE THEOREMS FOR STATIC OPTIMIZATION AND CALCULUS OF VARIATIONS. 2021. hal-03323994

\section{HAL Id: hal-03323994 \\ https://hal-paris1.archives-ouvertes.fr/hal-03323994}

Preprint submitted on 23 Aug 2021

HAL is a multi-disciplinary open access archive for the deposit and dissemination of scientific research documents, whether they are published or not. The documents may come from teaching and research institutions in France or abroad, or from public or private research centers.
L'archive ouverte pluridisciplinaire HAL, est destinée au dépôt et à la diffusion de documents scientifiques de niveau recherche, publiés ou non, émanant des établissements d'enseignement et de recherche français ou étrangers, des laboratoires publics ou privés. 


\title{
ENVELOPE THEOREMS FOR STATIC OPTIMIZATION AND CALCULUS OF VARIATIONS
}

\author{
JOËL BLOT, HASAN YILMAZ
}

\begin{abstract}
We establish differentiability properties of the value function of problems of Static Optimization in an abstract infinite dimensional setting and we apply that to problems of Calculus of Variations. We lighten the assumptions of existing results, notably by using Gâteaux and Hadamard differentials. Moreover we use recently established Multipliers Rules.
\end{abstract}

Key Words. Envelope theorem, Static optimization, Calculus of Variations. MSC 2020. 90C31, 90C30, 49J50.

\section{INTRODUCTION}

The paper treats of envelope theorems for parameterized problems of Static Optimization as

$$
(\mathcal{M}, \pi)\left\{\begin{aligned}
\text { Maximize } & f(x, \pi) \\
\text { subject to } & x \in \mathbb{G} \\
& \forall i=1, \ldots, k, g_{i}(x, \pi) \geq 0 \\
& \forall j=1, . ., \ell, h_{j}(x, \pi)=0,
\end{aligned}\right.
$$

and for parameterized problems of Calculus of Variations (Lagrange problems) under parameterized constraints as

$$
(\mathcal{V}, \pi)\left\{\begin{aligned}
\text { Maximize } & J(x, \pi):=\int_{0}^{T} L\left(t, x(t), x^{\prime}(t), \pi\right) d t \\
\text { subject to } & x \in C^{1}([0, T], M), x(0)=a_{0}, x(T)=a_{T} \\
& \forall i=1, \ldots, k, G_{i}(x, \pi):=\int_{0}^{T} \mathfrak{g}_{i}\left(t, x(t), x^{\prime}(t), \pi\right) d t \geq 0 \\
& \forall j=1, \ldots, \ell, H_{j}(x, \pi):=\int_{0}^{T} \mathfrak{h}_{j}\left(t, x(t), x^{\prime}(t), \pi\right) d t=0 .
\end{aligned}\right.
$$

We precise all the elements of these problems in the following sections.

We denote by $V(\pi)$ the value function of one of these problems. An envelope theorem consists to provide conditions to ensure the differentiability of $V$ (in a meaning that we will specify) and to provide an expression of this differential.

When $\pi_{0}$ is a fixed value of the parameter, in an envelope theorem, it is assumed that the optimal solution exists for all the parameters which belong to a neighborhood of $\pi_{0}$, not only for $\pi_{0}$. This is the difference between the problem of the envelope theorem and the problem of the regular perturbations where the theory provides conditions to ensure the existence of solutions $x(\pi)$ when $\pi$ is near to $\pi_{0}$, 4.

The envelope theorems are classical fundamental tools of the economic theory; cf. [5], 7], 8], 11], 17, 20, 21], 24], 25], 22] for example.

Our contribution is to establish envelope theorems with lightened assumptions with respect to the existing results. Notably we don't assume the regularity of the

Date: August, 23, 2021. 
multipliers which are associated to the solutions of these optimization problems, and we prove their continuity; their differentiability is unnecessary. We use the Hadamard differentiability instead of the continuous Fréchet differentiability that is weaker in infinite dimension. Note that to avoid to assume that the multipliers are of class $C^{1}$ is labelled as a conjecture in [5] (p. 7).

To realize this aim, we use recent multipliers rules established in 26] which contains an improvment of the results of 2 and an improvment of clasical results in the setting Fréchet $C^{1}$ as exposed in [1, we establish new results on the functionals under an integral form, we provide a variation of the Gram-Schmidt method, we provide informations on the dual space of an useful subspace of the space of the continuously differentiable functions defined on a segment of $\mathbb{R}$.

Now we describe the contents of the paper. In Section 2, we specify our notation, and we provide some preliminaries. In Section 3, we state an envelope theorem for the parameterized problems of Static Optimization in infinite dimension.

In Section 4, we prove the results of Section 3. In a first subsection, we establish results of topological algebra, we built a variation of the Gram-Schmidt method to permit us to prove the continuity of the multipliers (instead to assume it).

In Section 5, we establish an envelope theorem for the parameterized problems of Calculus of Variations. In a first subsection, we state an envelope theorem. In a second subsection, we establish new results on the differentiability of nonlinear integral functionals. In a third subsection, we establish results on the Euler equation. In a fourth subsection, we study dual spaces which are useful to our results, In the last subsection, we give a proof of our envelope theorem.

\section{Notation And Preliminaries}

$\mathbb{X}$ and $\mathbb{Y}$ are two real normed spaces.

2.1. Notions of differentiability. $\mathcal{L}(\mathbb{X}, \mathbb{Y})$ denotes the space of the continuous linear mappings from $\mathbb{X}$ into $\mathbb{Y}$. The topological dual space of $\mathbb{X}$ is denoted by $\mathbb{X}^{*}$. Generally the norm of the dual spaces will be denoted by $\|\cdot\|_{*}$. When $\mathbb{A} \subset \mathbb{X}$, $C^{0}(\mathbb{A}, \mathbb{Y})$ denotes the space of the continuous mappings from $\mathbb{A}$ into $\mathbb{Y}$.

Let $\mathbb{G}$ be an open subset of $\mathbb{X}, f: \mathbb{G} \rightarrow \mathbb{Y}$ be a mapping, $x \in \mathbb{G}$ and $v \in \mathbb{X}$. When it exists, the right-directional derivative (also called the right Gâteaux variation) of $f$ at $x$ in the direction $v$ is $D_{G}^{+} f(x ; v):=\lim _{\theta \rightarrow 0+} \frac{1}{\theta}(f(x+\theta v)-f(x))$. When $D_{G}^{+} f(x ; v)$ exists for all $v \in \mathbb{X}$ and when $D_{G}^{+} f(x ; \cdot) \in \mathcal{L}(\mathbb{X}, \mathbb{Y})$, the Gâteaux differential of $f$ at $x$ is $D_{G} f(x) \in \mathcal{L}(\mathbb{X}, \mathbb{Y})$ defined by $D_{G} f(x) \cdot v:=D_{G}^{+} f(x ; v)$.

We say that $f$ possesses a Hadamard variation at $x$ for the increment $v$ when there exists $D_{H}^{+} f(x ; v) \in \mathbb{Y}$ such that, for all $\left.\left(\theta_{n}\right)_{n} \in\right] 0,+\infty\left[{ }^{\mathbb{N}}\right.$ converging to 0 , and for all $\left(v_{n}\right)_{n} \in \mathbb{X}^{\mathbb{N}}$ converging to $v$, we have $\lim _{n \rightarrow+\infty} \frac{1}{\theta_{n}}\left(f\left(x+\theta_{n} v_{n}\right)-f(x)\right)=D_{H}^{+} f(x ; v)$. When $D_{H}^{+} f(x ; v)$ exists for all $v \in \mathbb{X}$, and when $D_{H}^{+} f(x ; \cdot) \in \mathcal{L}(\mathbb{X}, \mathbb{Y})$, the Hadamard differential of $f$ at $x$ is $D_{H} f(x) \in \mathcal{L}(\mathbb{X}, \mathbb{Y})$ defined by $D_{H} f(x) \cdot v:=D_{H}^{+} f(x ; v)$. When it exists, $D_{F} f(x)$ denotes the Fréchet differential of $f$ at $x . C^{1}(\mathbb{G}, \mathbb{Y})$ denotes the space of the continuously Fréchet differentiable mappings from $\mathbb{G}$ into $\mathbb{Y}$. When $T \in] 0,+\infty\left[, C^{1}([0, T], \mathbb{G})\right.$ denotes the space of the continuously differentiable functions from $[0, T]$ into $\mathbb{G}$, and when $\xi_{0}, \xi_{T} \in \mathbb{X}, C_{\xi_{0}, \xi_{T}}^{1}([0, T], \mathbb{G})$ denotes the space of the functions $x \in C^{1}([0, T], \mathbb{G})$ such that $x(0)=\xi_{0}$ and $x(T)=\xi_{T} . C_{c}^{\infty}\left([0, T], \mathbb{R}^{n}\right)$ denotes the space of the infinitely differentiable functions from $[0, T]$ into $\mathbb{R}^{n}$ such 
that their support is included into $] 0, T[$.

When $\mathbb{X}$ is a finite product of $m$ real normed spaces, $\mathbb{X}=\prod_{1<i<m} \mathbb{X}_{i}$, if $k \in$ $\{1, \ldots, m\}, D_{F, k} f(x)$ (respectively $D_{H, k} f(x)$, respectively $D_{G, k} f(x)$ ) denotes the partial Fréchet (respectively Hadamard, respectively Gâteaux) differential of $f$ at $x$ with respect to the $k$-th vector variable. If $1 \leq k_{1} \leq k_{2} \leq k_{3} \leq m, D_{H,\left(k_{1}, k_{2}, k_{3}\right)} f(x)$ denotes the Hadamard differential of the mapping

$\left[\left(x_{k_{1}}, x_{k_{2}}, x_{k_{3}}\right) \mapsto f\left(x_{1}, . ., x_{k_{1}}, \ldots, x_{k_{2}}, \ldots, x_{k_{3}}, \ldots, x_{m}\right)\right]$ at the point $\left(x_{k_{1}}, x_{k_{2}}, x_{k_{3}}\right)$. We refer to [10] for all these notions of differentiability.

2.2. Bounded variation functions. We consider the functions from $[0, T]$ into a normed vector space which are of bounded variation (cf. [19] (Chapter 10, Section 1). $B V([0, T], \mathbb{X})$ denotes the set of such functions. $C_{R}^{0}(] 0, T[, \mathbb{X})$ denotes the set of the functions from $[0, T]$ into $\mathbb{X}$ which are right-continuous on $] 0, T[$. We define $N B V([0, T], \mathbb{X}):=\left\{g \in B V([0, T], \mathbb{X}) \cap C_{R}^{0}(] 0, T[, \mathbb{X}): g(0)=0\right\}$. A function of $N B V([0, T], \mathbb{X})$ is called a normalized bounded variation function. $\|g\|_{B V}:=V_{0}^{T}(g)$ defines a norm on $N B V([0, T], \mathbb{X})$. If $\mathbb{X}$ is finite dimensional, if $\left(e_{i}\right)_{1 \leq i \leq d}$ is a basis of $\mathbb{X}$, and $\left(e_{i}^{*}\right)_{1 \leq i \leq d}$ is its dual basis, $g \in N B V([0, T], \mathbb{X})$ if and only if $e_{i}^{*} \circ g \in$ $N B V([0, T], \mathbb{R})$, and so we can use the results on scalar-valued bounded variation functions as given in [16], 15]. When $d=\operatorname{dim} \mathbb{X}<+\infty, f \in A C([a, b], \mathbb{X})(\mathrm{AC}$ means: absolutely continuity) if and only if $e_{i}^{*} \circ f \in A C([a, b], \mathbb{R})$ for all $i \in\{1, \ldots, d\}$, and so we can use the results on the scalar-valued absolutely continuous functions as given in [16, 6].

2.3. Integrals. When $\mathbb{E}$ is a real Banach space, $a<b$ in $\mathbb{R}$, and $f:[a, b] \rightarrow \mathbb{E}$ is a function, the Riemann integral of $f$ on $[a, b]$ is written $\int_{a}^{b} f(t) d t$ (cf. [9], chapter $8)$.

We denote by $\mathcal{B}([a, b])$ the Borel tribe of $[a, b]$, and by $\mathfrak{m}_{1}$ the canonical positive measure of Borel on $\mathcal{B}([a, b])$, charactarized by $\mathfrak{m}_{1}([\alpha, \beta[)=\beta-\alpha$ when $\alpha<\beta$ belong to $[a, b]$. When $f:[a, b] \rightarrow \mathbb{R}$ is a Borel function which is $\mathfrak{m}_{1}$-integrable, we say that $f$ is Borel integrable on $[a, b]$, and we denote its Borel integral by $\int_{[a, b]} f d \mathfrak{m}_{1}$. We denote the set of such functions by $\mathcal{L}^{1}\left([a, b], \mathcal{B}([a, b]), \mathfrak{m}_{1} ; \mathbb{R}\right)$. Conformly to 23, we don't use the term "Lebesgue integral" since we don't use the (completed) Lebesgue tribe, but only the Borel tribe.

When $\mathbb{E}$ is finite dimensional, if $\left(e_{i}\right)_{1 \leq i \leq d}$ is a basis of $\mathbb{E}$, and $\left(e_{i}^{*}\right)_{1 \leq i \leq d}$ its dual basis, a function $f:[a, b] \rightarrow \mathbb{E}$ is said to be Borel integrable on $[a, b]$ if and only if $e_{i}^{*} \circ f \in \mathcal{L}^{1}\left([a, b], \mathcal{B}([a, b]), \mathfrak{m}_{1} ; \mathbb{R}\right)$ for all $i \in\{1, \ldots, n\}$. We denote by $\mathcal{L}^{1}\left([a, b], \mathcal{B}([a, b]), \mathfrak{m}_{1} ; \mathbb{E}\right)$ the space of such functions.

\section{Static Optimization}

Let $\mathbb{X}$ and $\mathbb{Y}$ be two real normed spaces, and $\mathbb{G}$ be an open subset of $\mathbb{X}$. We consider the following functions $f: \mathbb{G} \times \mathbb{Y} \rightarrow \mathbb{R}, g_{i}: \mathbb{G} \times \mathbb{Y} \rightarrow \mathbb{R}$ for all $i \in\{1, \ldots, k\}$, and $h_{j}: \mathbb{G} \times \mathbb{Y} \rightarrow \mathbb{R}$ for all $j \in\{1, \ldots, \ell\}$. We denote by $V(\pi)$ the value of the problem $(\mathcal{M}, \pi)$ when $\pi \in \mathbb{Y}$.

Let $\pi_{0} \in \mathbb{Y}$. We consider the following conditions.

Condition on the dual space $\mathbb{X}^{*}$.

(ADUA) There exists $(\cdot \mid \cdot)_{\mathbb{X}^{*}} \in C^{0}\left(\mathbb{X}^{*} \times \mathbb{X}^{*}, \mathbb{R}\right)$ which is an inner product on $\mathbb{X}^{*}$. Conditions on the solutions. 
(Asol1) There exists an open neighborhood $P$ of $\pi_{0}$ in $\mathbb{Y}$ such that, for all $\pi \in P$, there exists a solution $\underline{x}(\pi)$ of $(\mathcal{M}, \pi)$.

(Asol2) There exists $\varpi \in \mathbb{Y}$ s.t. $D_{G}^{+} \underline{x}\left(\pi_{0} ; \varpi\right)$ exists.

(Asol2-bis) $\forall \varpi \in \mathbb{Y}, D_{G}^{+} \underline{x}\left(\pi_{0} ; \varpi\right)$ exists.

(Asol2-ter) $\forall \pi \in P, \forall \varpi \in \mathbb{Y}, D_{G}^{+} \underline{x}(\pi ; \varpi)$ exists.

Conditions on the functions of the criterion and of the constraints.

(AFOn1) $\forall \psi \in\{f\} \cup\left\{g_{i}: 1 \leq i \leq k\right\} \cup\left\{h_{j}: 1 \leq j \leq \ell\right\}, D_{H} \psi\left(\underline{x}\left(\pi_{0}\right), \pi_{0}\right)$ exists, and, for all $\pi \in P, D_{H, 1} \psi(\underline{x}(\pi), \pi)$ exists and $\left[\pi \mapsto D_{H, 1} \psi(\underline{x}(\pi), \pi)\right]$ is continuous on $P$.

(Afon2) $\forall \psi \in\{f\} \cup\left\{g_{i}: 1 \leq i \leq k\right\} \cup\left\{h_{j}: 1 \leq j \leq \ell\right\}, \forall \pi \in P, D_{H} \psi(\underline{x}(\pi), \pi)$ exists and $\left[\pi \mapsto D_{H, 2} \psi(\underline{x}(\pi), \pi)\right]$ is continuous on $P$.

Conditions on the constraints functions only.

(ACON1) $\forall \pi \in P, \forall j \in\{1, \ldots, \ell\},\left[x \mapsto h_{j}(x, \pi)\right]$ is continuous on a neighborhood of $\underline{x}(\pi)$.

(ACON2) $D_{H, 1} g_{1}\left(\underline{x}\left(\pi_{0}\right), \pi_{0}\right), \ldots, D_{H, 1} g_{k}\left(\underline{x}\left(\pi_{0}\right), \pi_{0}\right), D_{H, 1} h_{1}\left(\underline{x}\left(\pi_{0}\right), \pi_{0}\right), \ldots$

$D_{H, 1} h_{\ell}\left(\underline{x}\left(\pi_{0}\right), \pi_{0}\right)$ are linearly independent.

Theorem 3.1. (Envelope Theorem). We assume that (ADUA), (ASOL 1$)$, (ASOL2), (AFON1), (ACON1), (ACON2) are fulfilled. Then the following assertions hold.

(i) The right derivative of the value function of $(\mathcal{M}, \pi)$ at $\pi_{0}$ in the direction $\varpi$ exists and $D_{G}^{+} V\left(\pi_{0} ; \varpi\right)=D_{H, 2} f\left(\underline{x}\left(\pi_{0}\right), \pi_{0}\right) \cdot \varpi+$ $\sum_{1 \leq i \leq k} \lambda_{i}\left(\pi_{0}\right) D_{H, 2} g_{i}\left(\underline{x}\left(\pi_{0}\right), \pi_{0}\right) \cdot \varpi+\sum_{1 \leq j \leq \ell} \mu_{j}\left(\pi_{0}\right) D_{H, 2} h_{j}\left(\underline{x}\left(\pi_{0}\right), \pi_{0}\right) \cdot \varpi$, where $\lambda_{1}\left(\pi_{0}\right), \ldots, \lambda_{k}\left(\pi_{0}\right), \mu_{1}\left(\pi_{0}\right), \ldots, \mu_{\ell}\left(\pi_{0}\right)$ are the Karush-Kuhn-Tucker multipliers associated to the solution $\underline{x}\left(\pi_{0}\right)$ of $\left(\mathcal{M}, \pi_{0}\right)$.

(ii) If in addition we assume (ASOL2-bis) instead of (ASOL2), then the value function is Gâteaux differentiable at $\pi_{0}$, and $D_{G} V\left(\pi_{0}\right)=D_{H, 2} f\left(\underline{x}\left(\pi_{0}\right), \pi_{0}\right)+$ $\sum_{1 \leq i \leq k} \lambda_{i}\left(\pi_{0}\right) D_{H, 2} g_{i}\left(\underline{x}\left(\pi_{0}\right), \pi_{0}\right)+\sum_{1 \leq j \leq \ell} \mu_{j}\left(\pi_{0}\right) D_{H, 2} h_{j}\left(\underline{x}\left(\pi_{0}\right), \pi_{0}\right)$.

(iii) If in addition we assume that (ASOL2-ter) and (AFON2) are fulfilled, then the value function is of class Fréchet $C^{1}$ on an open neighborhood $Q$ of $\pi_{0}$, and for all $\pi \in Q$, we have $D_{F} V(\pi)=D_{H, 2} f(\underline{x}(\pi), \pi)+$

$\sum_{1 \leq i \leq k} \lambda_{i}(\pi) D_{H, 2} g_{i}(\underline{x}(\pi), \pi)+\sum_{1 \leq j \leq \ell} \mu_{j}(\pi) D_{H, 2} h_{j}(\underline{x}(\pi), \pi)$,

where $\lambda_{1}(\pi), \ldots, \lambda_{k}(\pi), \mu_{1}(\pi), \ldots, \mu_{\ell}(\pi)$ are the Karush-Kuhn-Tucker multipliers associated to the solution $\underline{x}(\pi)$ of $(\mathcal{M}, \pi)$.

We can adapt this result to problems where the domains of the functions are affine sets instead of to be vector spaces. Now we consider an affine subset $\mathbb{A} \subset \mathbb{X}$. The director vector subspace of $\mathbb{A}$ is denoted by $\mathbb{S}$. $\mathbb{G}^{0}$ is an open subset of $\mathbb{A}$. We consider the following functions. $f^{0}: \mathbb{G}^{0} \times \mathbb{Y} \rightarrow \mathbb{R}, g_{i}^{0}: \mathbb{G}^{0} \times \mathbb{Y} \rightarrow \mathbb{R}$ when $i \in\{1, \ldots, k\}$, and $h_{j}^{0}: \mathbb{G}^{0} \times \mathbb{Y} \rightarrow \mathbb{R}$ when $j \in\{1, \ldots, \ell\}$. With these elements, when $\pi \in \mathbb{Y}$, we build the following problem.

$$
\left(\mathcal{M}^{0}, \pi\right)\left\{\begin{aligned}
\text { Maximize } & f^{0}(z, \pi) \\
\text { subject to } & z \in \mathbb{G}^{0} \\
& \forall i=1, \ldots, k, g_{i}^{0}(z, \pi) \geq 0 \\
& \forall j=1, . ., \ell, h_{j}^{0}(z, \pi)=0
\end{aligned}\right.
$$


$V^{0}(\pi)$ denotes the value of $\left(\mathcal{M}^{0}, \pi\right)$. Let $\pi_{0} \in \mathbb{Y}$. We consider the following list of conditions.

Condition on the dual space $\mathbb{S}^{*}$.

$\left(A^{0}\right.$ DUA $)$ There exists $(\cdot \mid \cdot)_{\mathbb{S}^{*}} \in C^{0}\left(\mathbb{S}^{*} \times \mathbb{S}^{*}, \mathbb{R}\right)$ which is an inner product on $\mathbb{S}^{*}$.

Conditions on the solutions.

$\left(A^{0}\right.$ SOL1) There exists an open neighborhood $P$ of $\pi_{0}$ in $\mathbb{Y}$ such that, for all $\pi \in P$, there exists a solution $\underline{z}(\pi)$ of $\left(\mathcal{M}^{0}, \pi\right)$.

$\left(A^{0} \operatorname{SOL} 2\right)$ There exists $\varpi \in \mathbb{Y}$ s.t. $D_{G}^{+} \underline{z}\left(\pi_{0} ; \varpi\right)$ exists.

( $A^{0}$ SOL2-bis $) \forall \varpi \in \mathbb{Y}, D_{G}^{+} \underline{z}\left(\pi_{0} ; \varpi\right)$ exists.

( $A^{0}$ SOL2-ter $) \forall \pi \in P, \forall \varpi \in \mathbb{Y}, D_{G}^{+} \underline{z}(\pi ; \varpi)$ exists.

Conditions on the functions of the criterion and of the constraints.

( $A^{0}$ FON1) $\forall \psi \in\left\{f^{0}\right\} \cup\left\{g_{i}^{0}: 1 \leq i \leq k\right\} \cup\left\{h_{j}^{0}: 1 \leq j \leq \ell\right\}, D_{H} \psi\left(\underline{z}\left(\pi_{0}\right), \pi_{0}\right)$ exists, and, for all $\pi \in P, D_{H, 1} \psi(\underline{z}(\pi), \pi)$ exists and $\left[\pi \mapsto D_{H, 1} \psi(\underline{z}(\pi), \pi)\right]$ is continuous on $P$.

( $\left.A^{0} \mathrm{FON} 2\right) \forall \psi \in\left\{f^{0}\right\} \cup\left\{g_{i}^{0}: 1 \leq i \leq k\right\} \cup\left\{h_{j}^{0}: 1 \leq j \leq \ell\right\}, \forall \pi \in P, D_{H} \psi(\underline{z}(\pi), \pi)$ exists and $\left[\pi \mapsto D_{H, 2} \psi(\underline{z}(\pi), \pi)\right]$ is continuous on $P$.

Conditions on the constraints functions only.

$\left(A^{0} \mathrm{CON} 1\right) \forall \pi \in P, \forall j \in\{1, \ldots, \ell\},\left[z \mapsto h_{j}^{0}(z, \pi)\right]$ is continuous on a neighborhood of $\underline{z}(\pi)$.

$\left(A^{0} \mathrm{CON} 2\right) D_{H, 1} g_{1}\left(\underline{z}\left(\pi_{0}\right), \pi_{0}\right), \ldots, D_{H, 1} g_{k}\left(\underline{z}\left(\pi_{0}\right), \pi_{0}\right), D_{H, 1} h_{1}\left(\underline{z}\left(\pi_{0}\right), \pi_{0}\right), \ldots$,

$D_{H, 1} h_{\ell}\left(\underline{z}\left(\pi_{0}\right), \pi_{0}\right)$ are linearly independent.

The differentials on the affine subset $\mathbb{A}$ are defined (when they exist) on the director vector subset $\mathbb{S}$ which is the tangent vector space at each point of $\mathbb{A}$.

Corollary 3.2. We assume that ( $\left.A^{0} \mathrm{DUA}\right),\left(A^{0} \mathrm{SOL} 1\right)$, ( $\left.A^{0} \mathrm{SOL} 2\right),\left(A^{0} \mathrm{FON} 1\right)$, $\left(A^{0} \mathrm{CON} 1\right),\left(A^{0} \mathrm{CON} 2\right)$ are fulfilled. Then the following assertions hold.

( $\alpha) D_{G}^{+} V^{0}\left(\pi_{0} ; \varpi\right)$ exists and there exists $\left(\lambda_{i}\left(\pi_{0}\right)\right)_{1 \leq i \leq k} \in \mathbb{R}_{+}^{k}$ and $\left(\mu_{j}\left(\pi_{0}\right)\right)_{1 \leq j \leq \ell} \in \mathbb{R}^{\ell}$ such that $D_{G}^{+} V^{0}\left(\pi_{0} ; \varpi\right)=D_{H, 2} f^{0}\left(\underline{z}\left(\pi_{0}\right), \pi_{0}\right) \cdot \varpi+$ $\sum_{1 \leq i \leq k} \lambda_{i}\left(\pi_{0}\right) D_{H, 2} g_{i}^{0}\left(\underline{z}\left(\pi_{0}\right), \pi_{0}\right) \cdot \varpi+\sum_{1 \leq j \leq \ell} \mu_{j}\left(\pi_{0}\right) D_{H, 2} h_{j}^{0}\left(\underline{z}\left(\pi_{0}\right), \pi_{0}\right) \cdot \varpi$.

( $\beta$ ) If in addition we assume ( $\left.A^{0} \mathrm{SOL} 2-b i s\right)$ instead ( $\left.A^{0} \mathrm{SOL} 2\right)$, the value function is Gâteaux differentiable at $\pi_{0}$ and we have $D_{G} V^{0}\left(\pi_{0}\right)=D_{H, 2} f^{0}\left(\underline{z}\left(\pi_{0}\right), \pi_{0}\right)$ $+\sum_{1 \leq i \leq k} \lambda_{i}\left(\pi_{0}\right) D_{H, 2} g_{i}^{0}\left(\underline{z}\left(\pi_{0}\right), \pi_{0}\right)+\sum_{1 \leq j \leq \ell} \mu_{j}\left(\pi_{0}\right) D_{H, 2} h_{j}^{0}\left(\underline{z}\left(\pi_{0}\right), \pi_{0}\right)$.

$(\gamma)$ If in addition we assume that ( $\left.A^{0} \mathrm{SOL} 2-t e r\right)$ and $\left(A^{0} \mathrm{FON} 2\right)$ are fulfilled, then the value function is of class Fréchet $C^{1}$ on an open neighborhood $Q$ of $\pi_{0}$, and, for all $\pi \in Q$, there exist $\left(\left(\lambda_{i}(\pi)\right)_{1 \leq i \leq k},\left(\mu_{j}(\pi)\right)_{1 \leq j \leq \ell}\right) \in \mathbb{R}_{+}^{k} \times \mathbb{R}^{\ell}$ s.t. $D_{F} V^{0}(\pi)=D_{H, 2} f^{0}(\underline{z}(\pi), \pi)+$ $\sum_{1 \leq i \leq k} \lambda_{i}(\pi) D_{H, 2} g_{i}^{0}(\underline{z}(\pi), \pi)+\sum_{1 \leq j \leq \ell} \mu_{j}(\pi) D_{H, 2} h_{j}^{0}(\underline{z}(\pi), \pi)$.

The following corollary only uses the Fréchet differentiability and is more easily accessible to users for concrete problems issued from modellings.

Corollary 3.3. In the setting of Theorem 3.1, under (ADUA), (ASOL1), (ASOL2ter), (ACON2), we assume that the following condition is fulfilled.

$[\pi \mapsto \underline{x}(\pi)]$ is continuous on $P$ and $\forall \psi \in\{f\} \cup\left\{g_{i}: 1 \leq i \leq k\right\} \cup\left\{h_{j}: 1 \leq j \leq \ell\right\}$, $\psi$ is Fréchet $C^{1}$ on $\mathbb{G} \times P$.

Then (AFON1), (AFON2), (ACON1) are fulfilled, and all the conclusions of Theorem 3.1 hold, replacing the Hadamard differentials by Fréchet differentials in the formulas. 
Remark 3.4. When $\mathbb{X}$ is finite dimensional, the conditions (ADUA) and ( $\left.A^{0} \mathrm{DUA}\right)$ are automatically fulfilled. Moreover the Hadamard differentiability is equivalent to the Fréchet differentiability. Hence, without (ADUA), the statement of Theorem 3.1 holds and we can replace the Hadamard differentials by Fréchet differentials in the formulas of the conclusions.

\section{Proofs of Results of SeCtion 3}

4.1. Results of topological algebra. $\mathbb{E}$ and $\mathbb{W}$ are two real normed spaces.

Lemma 4.1. Let $w_{0} \in \mathbb{W}$ and $W$ be a neighborhood of $w_{0}$ in $\mathbb{W}$. Let $\left(b_{i}\right)_{1 \leq i \leq m} \in$ $C^{0}(W, \mathbb{E})^{m}$ s.t. $b_{1}\left(w_{0}\right), \ldots, b_{m}\left(w_{0}\right)$ are linearly independent. Then there exists a neighborhood $W_{0}$ of $w_{0}, W_{0} \subset W$, s.t. for all $w \in W_{0}, b_{1}(w), \ldots, b_{m}(w)$ are linearly independent.

Proof. For all $w \in W$, we consider the mapping $\Phi_{w}: \mathbb{R}^{m} \rightarrow \mathbb{E}$ defined by $\Phi_{w}\left(u^{1}, \ldots, u^{m}\right):=\sum_{1 \leq i \leq m} u^{i} b_{i}(w)$ when $\left(u^{1}, \ldots, u^{m}\right) \in \mathbb{R}^{m}$. Clearly we have $\Phi_{w} \in$ $\mathcal{L}\left(\mathbb{R}^{m}, \mathbb{E}\right)$, and the linear independence of $b_{1}(w), \ldots, b_{m}(w)$ is equivalent to have $\operatorname{Ker} \Phi_{w}=\{0\}$. This last equality is equivalent to have $\Phi_{w}^{-1}(\{0\}) \cap S(0,1)=\{0\}$, where $S(0,1)$ is the unit sphere of $\mathbb{R}^{m}$. Proceeding by contradiction, we assume: $\forall k \in \mathbb{N}_{*}, \exists w_{k} \in B\left(w_{0}, \frac{1}{k}\right), \exists v_{k} \in S(0,1)$ s.t. $\Phi_{w_{k}}\left(v_{k}\right)=0$. Using the compactness of $\mathrm{S}(0,1)$ and the Weierstras-Bolzano theorem, we obtain the existence of an increasing function $\sigma: \mathbb{N}_{*} \rightarrow \mathbb{N}_{*}$ and of $u \in S(0,1)$ s.t. $\lim _{k \rightarrow+\infty} v_{\sigma(k)}=u$. Hence, we have, for all $k \in \mathbb{N}_{*}, \sum_{1 \leq i \leq m} v_{\sigma(k)}^{i} b_{i}\left(w_{\sigma(k)}\right)=0$. Since the $b_{i}$ are continuous, we obtain $\sum_{1 \leq i \leq m} u^{i} b_{i}\left(w_{0}\right)=0$, and the linear independence of the $b_{i}\left(w_{0}\right)$ implies that $u=0$ which contredicts $u \in S(0,1)$.

In the two following lemmas, we assume the existence of functional $(\cdot \mid \cdot)_{\mathbb{E}} \in$ $C^{0}(\mathbb{E} \times \mathbb{E}, \mathbb{R})$ which is an inner product on $\mathbb{E}$. The following lemma is a version of the Gram-Schmidt's process (cf. e.g. 18] p. 366) in presence of a parameter.

Lemma 4.2. Let $W$ be an open subset of $\mathbb{W}$, and $\left(e_{i}\right)_{1 \leq i \leq n} \in C^{0}(W, \mathbb{E})^{n}$ s.t. $e_{1}(w)$, $\ldots, e_{n}(w)$ are linearly independent for all $w \in W$.

Then there exists $\left(\epsilon_{i}\right)_{1 \leq i \leq n} \in C^{0}(W, \mathbb{E} \backslash\{0\})^{n}$ s.t. the following properties hold.

(a) $\forall w \in W, \forall i \in\{1, \ldots n\}, \epsilon_{i}(w) \in \operatorname{span}\left\{e_{1}(w), \ldots, e_{i}(w)\right\}$.

(b) $\forall w \in W, \forall(i, j) \in\{1, \ldots n\}^{2}$ s.t. $i \neq j,\left(\epsilon_{i}(w) \mid \epsilon_{j}(w)\right)_{\mathbb{E}}=0$.

(c) $\forall(i, j) \in\{1, \ldots n\}^{2}, \exists \alpha_{j}^{i} \in C^{0}(W, \mathbb{R})$ s.t., $\forall w \in W$, $\epsilon_{j}(w)=\sum_{1 \leq i \leq n} \alpha_{j}^{i}(w) e_{i}(w)$, where $\alpha_{j}^{j}(w)=1$ and $\alpha_{j}^{i}(w)=0$ when $i>j$.

Proof. We proceed by induction on $n$. When $n=1$, we set $\epsilon_{1}:=e_{1}$, and we have $\alpha_{1}^{1}(w)=1$ for all $w \in W$, and the conclusions (a, b, c) are fulfilled. We do the assumption of induction on $k$, and we prove the result for $k+1$. We set

$$
\epsilon_{k+1}(w):=e_{k+1}(w)-\sum_{1 \leq i \leq k} \frac{\left(e_{k+1}(w) \mid \epsilon_{i}(w)\right)_{\mathbb{E}}}{\left\|\epsilon_{i}(w)\right\|_{e}^{2}} \epsilon_{i}(w)
$$

where $\|\cdot\|_{e}$ is the norm associated to $(\cdot \mid \cdot)_{\mathbb{E}}$.

Hence $\epsilon_{k+1}(w) \in \operatorname{span}\left\{e_{k+1}(w), \epsilon_{1}(w), \ldots, \epsilon_{k}(w)\right\}=\operatorname{span}\left\{e_{j}(w): 1 \leq j \leq k+1\right\}$ by using the induction assumption, and so (a) is fulfilled. Let $i<k+1$. We have $\left(\epsilon_{k+1}(w) \mid \epsilon_{i}(w)\right)_{\mathbb{E}}=\left(e_{k+1}(w) \mid \epsilon_{i}(w)\right)_{\mathbb{E}}$

$-\sum_{1 \leq j \leq k, j \neq i} \frac{\left(e_{k+1}(w) \mid \epsilon_{j}(w)\right)_{\mathbb{E}}}{\left\|\epsilon_{j}(w)\right\|_{e}^{2}}\left(\epsilon_{j}(w) \mid \epsilon_{i}(w)\right)_{\mathbb{E}}-\frac{\left(e_{k+1}(w) \mid \epsilon_{i}(w)\right)_{\mathbb{E}}}{\left\|\epsilon_{i}(w)\right\|_{e}^{2}}\left(\epsilon_{i}(w) \mid \epsilon_{i}(w)\right)_{\mathbb{E}}$

$=\left(e_{k+1}(w) \mid \epsilon_{i}(w)\right)_{\mathbb{E}}-\left(e_{k+1}(w) \mid \epsilon_{i}(w)\right)_{\mathbb{E}}=0$. 
Hence, (b) is fulfilled. Notice that $\epsilon_{k+1}(w) \neq 0$ for all $w \in W$, since, proceeding by contradiction, if there exists $w \in W$ s.t. $\epsilon_{k+1}(w)=0$, then, using (4.1), we obtain $e_{k+1}(w) \in \operatorname{span}\left\{e_{i}(w): 1 \leq i \leq k\right\}$ which contradicts the linear independence of the $e_{i}(w)$. When $j \in\{1, \ldots, k\}$, we inductively define

$$
\alpha_{k+1}^{j}(w):=-\sum_{1 \leq i \leq k} \frac{\left(e_{k+1}(w) \mid \epsilon_{i}(w)\right)_{\mathbb{E}}}{\left\|\epsilon_{i}(w)\right\|_{e}^{2}} \alpha_{i}^{j}(w), \quad \alpha_{k+1}^{k+1}(w):=1
$$

and, when $j>k+1$, we set

$$
\alpha_{k+1}^{j}(w):=0 .
$$

Since $(\cdot \mid \cdot)_{\mathbb{E}},\|\cdot\|_{e}^{2}, e_{1}, \ldots, e_{k}, \epsilon_{1}, \ldots, \epsilon_{k}$ are continuous, we see that $\alpha_{k+1}^{j}$ is continuous as a composition of continuous mappings, for all $j$. Doing a straightforward calculation, we obtain

$$
\epsilon_{k+1}(w)=\sum_{1 \leq j \leq k+1} \alpha_{k+1}^{j}(w) e_{j}(w)=\sum_{1 \leq j \leq n} \alpha_{k+1}^{j}(w) e_{j}(w) .
$$

Hence the reasoning by induction is complete, and the proof of the lemma is complete.

Lemma 4.3. Let $W$ be an open subset of $\mathbb{W}$ and $\left(e_{i}\right)_{1 \leq i \leq n} \in C^{0}(W, \mathbb{E})^{n}$ s.t. $e_{1}(w)$, $\ldots, e_{n}(w)$ are linearly independent, for all $w \in W$. We set $F_{w}:=\operatorname{span}\left\{e_{i}(w): 1 \leq\right.$ $i \leq n\}$ for all $w \in W$, and $F:=\bigcup_{w \in W}\left(F_{w} \times\{w\}\right)$. When $(x, w) \in F, \mathfrak{x}^{i}(x, w)$ denotes the $i$-th coordinate of $x$ in the basis $\left(e_{j}(w)\right)_{1 \leq j \leq n}$. Then, for all $i \in\{1, \ldots, n\}, \mathfrak{x} i \in C^{0}(F, \mathbb{R})$.

Proof. We consider $\left(\epsilon_{j}\right)_{1 \leq j \leq n} \in C^{0}(W, \mathbb{E})^{n}$ as defined in Lemma 4.2. When $w \in W$ and $x \in F_{w}$, we can write $x=\sum_{1 \leq i \leq n} y^{i}(x, w) \epsilon_{i}(w)$ where $y^{i}(x, w):=\frac{\left(x \mid \epsilon_{i}(w)\right)_{\mathbb{E}}}{\left\|\epsilon_{i}(w)\right\|_{e}^{2}}$. Using Lemma 4.2 we know that $\epsilon_{i} \in C^{0}(W, \mathbb{E} \backslash\{0\})$, and using the continuity of $(\cdot \mid \cdot)_{\mathbb{E}}$ with respect to the intial norm of $\mathbb{E} \times \mathbb{E}$, we obtain that $y^{i} \in C^{0}(F, \mathbb{R})$ as a composition of continuous mappings.

Since $\left(e_{i}(w)\right)_{1 \leq i \leq n}$ and $\left(\epsilon_{i}(w)\right)_{1 \leq i \leq n}$ are two bases of the same vector space $F_{w}$, we can consider $M(w)$ the matrix of transition from $\left(e_{i}(w)\right)_{1 \leq i \leq n}$ to $\left(\epsilon_{i}(w)\right)_{1 \leq i \leq n}$. Using Lemma 4.2, the elements of the $i$-th column of $M(w)$ are: $\alpha_{1}^{i}(w), \ldots, \alpha_{i-1}^{i}$, $1,0, \ldots, 0$, and since the elements are continuous functions of $w$, we can assert that $[w \mapsto M(w)] \in C^{0}(W, G L(n, \mathbb{R}))$ where $G L(n, \mathbb{R})$ is the classical group on the $n \times n$ invertible real matrices. The operator $\mathcal{I}: G L(n, \mathbb{R}) \rightarrow G L(n, \mathbb{R})$, defined by $\mathcal{I}(L):=L^{-1}$, is well know to be continuous. Moreover $M(w)^{-1}$ is the matrix of transition from $\left(\epsilon_{i}(w)\right)_{1 \leq i \leq n}$ to $\left(e_{i}(w)\right)_{1 \leq i \leq n}$. Since we have $\left[\mathfrak{x}^{1}(x, w) \ldots \mathfrak{x}^{n}(x, w)\right]^{t}=$ $M(w)^{-1}\left[y^{1}(x, w) \ldots y^{n}(x, w)\right]^{t}$, where the upper index $t$ denotes the transposition of the matrices, we obtain the continuity of the $\mathfrak{x}^{i}$ on $F$ as a composition of continuous mappings.

4.2. Proof of Theorem 3.1. Conclusion (i). We use Lemma 4.1 with $\mathbb{W}=\mathbb{Y}$, $\mathbb{E}=\mathbb{X}^{*}$ and (ACON2) to ensure the existence of an open neighborhood $Q$ of $\pi_{0}$ in $P$ such that,

$$
\left.\begin{array}{l}
\forall \pi \in Q, D_{H, 1} g_{1}(\underline{x}(\pi), \pi), \ldots, D_{H, 1} g_{k}(\underline{x}(\pi), \pi), D_{H, 1} h_{1}(\underline{x}(\pi), \pi), \\
\ldots, D_{H, 1} h_{\ell}(\underline{x}(\pi), \pi) \text { are linearly independent. }
\end{array}\right\}
$$

We want to use the multipliers rule of [26] (Theorem 2.2). Notice that our assumption (AFON1) implies that assumptions (i) and (ii) of this multipliers rule are 
fulfilled. Our assumption (Afon1) and Theorem 4.2.6 in [10] imply that the third assumption of this multipliers rule is fulfilled. (ACON1) and (AFON1) imply that the last assumption of this multipliers rule is fulfilled. Hence using this multipliers rule and (4.5), we can ensure the existence of multipliers $\left(\lambda_{i}(\pi)\right)_{1 \leq i \leq k} \in \mathbb{R}^{k}$ and $\left(\mu_{j}(\pi)\right)_{1 \leq j \leq \ell} \in \mathbb{R}^{\ell}$ which satisfy the following conditions for all $\pi \in Q$.

$$
\left.\begin{array}{c}
\forall \pi \in Q, \forall i \in\{1, \ldots, k\}, \lambda_{i}(\pi) \geq 0 . \\
\forall \pi \in Q, \forall i \in\{1, \ldots, k\}, \lambda_{i}(\pi) g_{i}(\underline{x}(\pi), \pi)=0 . \\
\forall \pi \in Q, \quad D_{H, 1} f(\underline{x}(\pi), \pi)+\sum_{1 \leq i \leq k} \lambda_{i}(\pi) D_{H, 1} g_{i}(\underline{x}(\pi), \pi) \\
+\sum_{1 \leq j \leq \ell} \mu_{j}(\pi) D_{H, 1} h_{j}(\underline{x}(\pi), \pi) \stackrel{=}{=} .
\end{array}\right\}
$$

The following result establishes the continuity of the Karush-Kuhn-Tucker multipliers with respect to the parameter $\pi$.

Lemma 4.4. For all $i \in\{1, \ldots, k\}, \lambda_{i} \in C^{0}\left(Q, \mathbb{R}_{+}\right)$and, for all $j \in\{1, \ldots, \ell\}$, $\mu_{j} \in C^{0}(Q, \mathbb{R})$.

Proof. Under (Afon1), (AduA), with $\mathbb{W}=\mathbb{Y}$ and $\mathbb{E}=\mathbb{X}^{*}$, we can use Lemma 4.3. we set $e_{1}(\pi):=D_{H, 1} g_{1}(\underline{x}(\pi), \pi), \ldots, e_{k}(\pi):=D_{H, 1} g_{k}(\underline{x}(\pi), \pi), e_{k+1}(\pi):=$ $D_{H, 1} h_{1}(\underline{x}(\pi), \pi), \ldots, e_{k+\ell}(\pi):=D_{H, 1} h_{\ell}(\underline{x}(\pi), \pi)$, and using (4.5) and (4.8), the assumptions of Lemma 4.3 are fulfilled, and we can conclude that the coordinates $\lambda_{i}$ and $\mu_{j}$ are continuous.

Since we have $V(\pi)=f(\underline{x}(\pi), \pi)$ for all $\pi \in Q$, and since the Hadamard differential satisfies the Chain Rule ([10], (4.2.5) p.263), we can calculate

$$
D_{G}^{+} V\left(\pi_{0} ; \varpi\right)=D_{H, 1} f\left(\underline{x}\left(\pi_{0}\right), \pi_{0}\right) \cdot D_{G}^{+} \underline{x}\left(\pi_{0} ; \varpi\right)+D_{H, 2} f\left(\underline{x}\left(\pi_{0}\right), \pi_{0}\right) \cdot \varpi .
$$

Under (4.8), we also have

$$
\left.\begin{array}{l}
D_{H, 1} f\left(\underline{x}\left(\pi_{0}\right), \pi_{0}\right) \cdot D_{G}^{+} \underline{x}\left(\pi_{0} ; \varpi\right)= \\
-\sum_{1 \leq i \leq k} \lambda_{i}\left(\pi_{0}\right) D_{H, 1} g_{i}\left(\underline{x}\left(\pi_{0}\right), \pi_{0}\right) \cdot D_{G}^{+} \underline{x}\left(\pi_{0} ; \varpi\right) \\
-\sum_{1 \leq j \leq \ell} \mu_{j}\left(\pi_{0}\right) D_{H, 1} h_{j}\left(\underline{x}\left(\pi_{0}\right), \pi_{0}\right) \cdot D_{G}^{+} \underline{x}\left(\pi_{0} ; \varpi\right) .
\end{array}\right\}
$$

If $\lambda_{i}\left(\pi_{0}\right)>0$, using Lemma 4.4, we can assert that there exists a neighborhood $N$ of $\pi_{0}$ in $Q$ such that $\lambda_{i}(\pi)>0$ for all $\pi \in N$, and using (4.7) we obtain $g_{i}(\underline{x}(\pi), \pi)=0$ for all $\pi \in N$. Differentiating with respect to $\pi$, we obtain

$$
D_{H, 1} g_{i}\left(\underline{x}\left(\pi_{0}\right), \pi_{0}\right) \cdot D_{G}^{+} \underline{x}\left(\pi_{0} ; \varpi\right)+D_{H, 2} g_{i}\left(\underline{x}\left(\pi_{0}\right), \pi_{0}\right) \cdot \varpi=0 .
$$

To subsume this reasoning, we write

$$
\left.\begin{array}{l}
\lambda_{i}\left(\pi_{0}\right)>0 \Longrightarrow \lambda_{i}\left(\pi_{0}\right) D_{H, 1} g_{i}\left(\underline{x}\left(\pi_{0}\right), \pi_{0}\right) \cdot D_{G}^{+} \underline{x}\left(\pi_{0} ; \varpi\right) \\
=-\lambda_{i}\left(\pi_{0}\right) D_{H, 2} g_{i}\left(\underline{x}\left(\pi_{0}\right), \pi_{0}\right) \cdot \varpi .
\end{array}\right\}
$$

Moreover the following assertion is clear.

$$
\left.\begin{array}{l}
\lambda_{i}\left(\pi_{0}\right)=0 \Longrightarrow \lambda_{i}\left(\pi_{0}\right) D_{H, 1} g_{i}\left(\underline{x}\left(\pi_{0}\right), \pi_{0}\right) \cdot D_{G}^{+} \underline{x}\left(\pi_{0} ; \varpi\right) \\
=-\lambda_{i}\left(\pi_{0}\right) D_{H, 2} g_{i}\left(\underline{x}\left(\pi_{0}\right), \pi_{0}\right) \cdot \varpi .
\end{array}\right\}
$$

From (4.11) and (4.12), we obtain

$$
\left.\begin{array}{l}
\forall i \in\{1, \ldots, k\}, \lambda_{i}\left(\pi_{0}\right) D_{H, 1} g_{i}\left(\underline{x}\left(\pi_{0}\right), \pi_{0}\right) \cdot D_{G}^{+} \underline{x}\left(\pi_{0} ; \varpi\right) \\
=-\lambda_{i}\left(\pi_{0}\right) D_{H, 2} g_{i}\left(\underline{x}\left(\pi_{0}\right), \pi_{0}\right) \cdot \varpi .
\end{array}\right\}
$$

Since $h_{j}(\underline{x}(\pi), \pi)=0$ when $\pi \in Q$, differentiating at $\pi_{0}$, we obtain the following assertion.

$$
\left.\begin{array}{l}
\forall j \in\{1, \ldots, \ell\}, \mu_{j}\left(\pi_{0}\right) D_{H, 1} h_{j}\left(\underline{x}\left(\pi_{0}\right), \pi_{0}\right) \cdot D_{G}^{+} \underline{x}\left(\pi_{0} ; \varpi\right) \\
=-\mu_{j}\left(\pi_{0}\right) D_{H, 2} h_{j}\left(\underline{x}\left(\pi_{0}\right), \pi_{0}\right) \cdot \varpi .
\end{array}\right\}
$$


Using (4.13) and (4.14) into (4.10), we have

$$
\left.\begin{array}{l}
D_{H, 1} f\left(\underline{x}\left(\pi_{0}\right), \pi_{0}\right) \cdot D_{G}^{+} \underline{x}\left(\pi_{0} ; \varpi\right)=\sum_{1 \leq i \leq k} \lambda_{i}\left(\pi_{0}\right) D_{H, 2} g_{i}\left(\underline{x}\left(\pi_{0}\right), \pi_{0}\right) \cdot \varpi \\
+\sum_{1 \leq j \leq \ell} \mu_{j}\left(\pi_{0}\right) D_{H, 2} h_{j}\left(\underline{x}\left(\pi_{0}\right), \pi_{0}\right) \cdot \varpi .
\end{array}\right\}
$$

Using (4.15) into (4.9), we obtain: $D_{G}^{+} V\left(\pi_{0} ; \varpi\right)=D_{H, 2} f\left(\underline{x}\left(\pi_{0}\right), \pi_{0}\right) \cdot \varpi$

$+\sum_{1 \leq i \leq k} \lambda_{i}\left(\pi_{0}\right) D_{H, 2} g_{i}\left(\underline{x}\left(\pi_{0}\right), \pi_{0}\right) \cdot \varpi+\sum_{1 \leq j \leq \ell} \mu_{j}\left(\pi_{0}\right) D_{H, 2} h_{j}\left(\underline{x}\left(\pi_{0}\right), \pi_{0}\right) \cdot \varpi$, and so the first conclusion of Theorem 3.1 is proven.

Conclusion (ii). Since (Asol2-bis) implies (Asol2), we can use the formula of $D_{G}^{+} V\left(\pi_{0}, \varpi\right)$ for all the increments $\varpi$; from this formula we see that

$\left[\varpi \mapsto D_{G}^{+} V\left(\pi_{0}, \varpi\right)\right]$, from $\mathbb{Y}$ into $\mathbb{R}$, is a linear combination of elements of $\mathbb{Y}^{*}$. Consequently $V$ is Gâteaux differentiable at $\pi_{0}$, and so the second conclusion of Theorem 3.1 is proven.

Conclusion (iii). From (Asol2-ter), using the second conclusion at $\pi \in Q$ instead of $\pi_{0}$, we know that, for all $\pi \in Q$, we have $D_{G} V(\pi)=D_{H, 2} f(\underline{x}(\pi), \pi)+$ $\sum_{1 \leq i \leq k} \lambda_{i}(\pi) D_{H, 2} g_{i}(\underline{x}(\pi), \pi)+\sum_{1 \leq j \leq \ell} \mu_{j}(\pi) D_{H, 2} h_{j}(\underline{x}(\pi), \pi)$.

Using (Afon2), we see that $D_{G} V \in C^{0}\left(Q, \mathbb{Y}^{*}\right)$, hence ([10], (4.4.7) Corollary 2, p.257), $V$ is Fréchet differentiable on $Q$ and $D_{F} V \in C^{0}\left(Q, \mathbb{Y}^{*}\right)$. The proof of Theorem 3.1 is complete.

4.3. Proof of Corollary 3.2. The strategy to realize this proof is to translate $\left(\mathcal{M}^{0}, \pi\right)$ into a new problem $\left(\mathcal{M}^{1}, \pi\right)$ which is a special case of $(\mathcal{M}, \pi)$, to apply Theorem 3.1 on $\left(\mathcal{M}^{1}, \pi\right)$, and to translate the conclusions on $\left(\mathcal{M}^{1}, \pi\right)$ into conclusions on $\left(\mathcal{M}^{0}, \pi\right)$.

For all $\psi^{0} \in\left\{f^{0}\right\} \cup\left\{g_{i}^{0}: 1 \leq i \leq k\right\} \cup\left\{h_{j}^{0}: 1 \leq j \leq \ell\right\}$ we introduce the function $\psi:\left(\mathbb{G}^{0}-\underline{z}\left(\pi_{0}\right)\right) \times \mathbb{Y} \rightarrow \mathbb{R}$ by setting $\psi(x, \pi):=\psi^{0}\left(x+\underline{z}\left(\pi_{0}\right), \pi\right)$. Note that $\mathbb{G}^{0}-\underline{z}\left(\pi_{0}\right)$ is an open subset of $\mathbb{S}$. We consider the new following problem.

$$
\left(\mathcal{M}^{1}, \pi\right) \begin{cases}\text { Maximize } & f(x, \pi) \\ \text { subject to } & x \in \mathbb{G}^{0}-\underline{z}\left(\pi_{0}\right) \\ & \forall i \in\{1, \ldots, k\}, g_{i}(x, \pi) \geq 0 \\ & \forall j \in\{1, \ldots, \ell\}, h_{j}(x, \pi)=0 .\end{cases}
$$

This problem is a special case of $(\mathcal{M}, \pi)$ with $\mathbb{X}=\mathbb{S}$. It is easy to verify that $x$ is admissible for $\left(\mathcal{M}^{1}, \pi\right)$ if and only if $x+\underline{z}\left(\pi_{0}\right)$ is admissible for $\left(\mathcal{M}^{0}, \pi\right)$, and $x$ is a solution of $\left(\mathcal{M}^{1}, \pi\right)$ if and only if $x+\underline{z}\left(\pi_{0}\right)$ is a solution of $\left(\mathcal{M}^{0}, \pi\right)$. We set $\underline{x}(\pi):=\underline{z}(\pi)-\underline{z}\left(\pi_{0}\right)$. After that, note that the assumptions on $\left(\mathcal{M}^{0}, \pi\right)$ were done to be translatable into assumptions of Theorem 3.1] on $\left(\mathcal{M}^{1}, \pi\right)$. Then the conclusions of Theorem 3.1 are valid on $\left(\mathcal{M}^{1}, \pi\right)$. Now it suffices to translate the conclusions on $\left(\mathcal{M}^{1}, \pi\right)$ into conclusions on $\left(\mathcal{M}^{0}, \pi\right)$. To do this, it suffices to note that $D_{G}^{+} \underline{x}(\pi ; \varpi)=D_{G}^{+} \underline{z}(\pi ; \varpi)$ and, for all $\psi^{0} \in\left\{f^{0}\right\} \cup\left\{g_{i}^{0}: 1 \leq i \leq k\right\} \cup\left\{h_{j}^{0}: 1 \leq\right.$ $j \leq \ell\}, D_{H, 2} \psi^{0}(\underline{z}(\pi), \pi)=D_{H, 2} \psi(\underline{x}(\pi), \pi)$. The proof of the corollary is complete.

\section{Calculus of Variations}

This section is divided into the following subsections. In a first subsection, we state an envelope theorem for $(\mathcal{V}, \pi)$ after to specify the assumptions. In a second subsection, we establish new results on functionals under an integral form. In a third subsection, we treat of the Euler-Lagrange equation. In the last subsection, we give a proof of the envelope theorem. 
5.1. An envelope theorem. $X=\mathbb{R}^{n}$ and $Y$ is a real normed space. $\left.T \in\right] 0,+\infty[$ and $M$ is an open subset of $\mathbb{R}^{n}$. We consider functions $L:[0, T] \times M \times \mathbb{R}^{n} \times Y \rightarrow \mathbb{R}$, $\mathfrak{g}_{i}:[0, T] \times M \times \mathbb{R}^{n} \times Y \rightarrow \mathbb{R}$ for all $i \in\{1, \ldots, k\}, \mathfrak{h}_{j}:[0, T] \times M \times \mathbb{R}^{n} \times Y \rightarrow \mathbb{R}$ for all $j \in\{1, \ldots, \ell\}$. We fix $a_{0}, a_{T} \in M$.

We fix $\pi_{0} \in Y$ and we consider the following list of conditions:

\section{Conditions on the solutions}

(Bsol1) There exists an open neighborhood $P$ of $\pi_{0}$ in $Y$ s.t., for all $\pi \in P$, there exists a solution $x(\pi)$ of $(\mathcal{V}, \pi)$, and $[\pi \mapsto x(\pi)]$ is continuous at $\pi_{0}$.

(Bsol2) There exists $\varpi \in Y$ s.t. $D_{G}^{+} x\left(\pi_{0} ; \varpi\right)$ exists.

(Bsol2-bis) For all $\varpi \in Y, D_{G}^{+} x\left(\pi_{0} ; \varpi\right)$ exists.

(Bsol2-ter) For all $\pi \in P$, for all $\varpi \in Y, D_{G}^{+} x(\pi ; \varpi)$ exists.

Conditions on the integrand of the criterion and of the constraints

(BINT1) There exist $\rho>0$ and $\xi \in \mathcal{L}^{1}\left([0, T], \mathcal{B}([0, T]), \mathbb{R}_{+}, \mathfrak{m}_{1}\right)$ s.t., for all $\psi \in\{L\} \cup$ $\left\{\mathfrak{g}_{i}: 1 \leq i \leq k\right\} \cup\left\{\mathfrak{h}_{j}: 1 \leq j \leq \ell\right\}$, for all $t \in[0, T]$, for all $u, u_{1} \in B\left(x\left(\pi_{0}\right)(t), \rho\right)$, for all $v, v_{1} \in B\left(x\left(\pi_{0}\right)^{\prime}(t), \rho\right)$, for all $\pi, \pi_{1} \in B\left(\pi_{0}, \rho\right)$,

$\left|\psi(t, u, v, \pi)-\psi\left(t, u_{1}, v_{1}, \pi_{1}\right)\right| \leq \xi(t)\left(\left\|u-u_{1}\right\|+\left\|v-v_{1}\right\|+\left\|\pi-\pi_{1}\right\|\right)$.

(BINT2) For all $\psi \in\{L\} \cup\left\{\mathfrak{g}_{i}: 1 \leq i \leq k\right\} \cup\left\{\mathfrak{h}_{j}: 1 \leq j \leq \ell\right\}$, $\psi$ is continuous on $[0, T] \times M \times \mathbb{R}^{n} \times P$, and for all $t \in[0, T]$, for all $\pi \in P$,

$D_{H,(2,3,4)} \psi\left(t, x(\pi)(t), x(\pi)^{\prime}(t), \pi\right)$ exists.

(BINT3) For all $\psi \in\{L\} \cup\left\{\mathfrak{g}_{i}: 1 \leq i \leq k\right\} \cup\left\{\mathfrak{h}_{j}: 1 \leq j \leq \ell\right\}$, for all $t \in[0, T]$, $\left[\pi \mapsto D_{H,(2,3)} \psi\left(t, x(\pi)(t), x(\pi)^{\prime}(t), \pi\right)\right]$ is continuous from $B\left(\pi_{0}, \rho\right)$ into $\left(\mathbb{R}^{n *},\|\cdot\|_{*}\right)^{2}$. (BINT3-bis) For all $\psi \in\{L\} \cup\left\{\mathfrak{g}_{i}: 1 \leq i \leq k\right\} \cup\left\{\mathfrak{h}_{j}: 1 \leq j \leq \ell\right\}$, for all $t \in[0, T]$, $\left[\pi \mapsto D_{H,(2,3,4)} \psi\left(t, x(\pi)(t), x(\pi)^{\prime}(t), \pi\right)\right]$ is continuous on $B\left(\pi_{0}, \rho\right)$.

(BINT4) For all $\psi \in\{L\} \cup\left\{\mathfrak{g}_{i}: 1 \leq i \leq k\right\} \cup\left\{\mathfrak{h}_{j}: 1 \leq j \leq \ell\right\}$, for all $\pi \in B\left(\pi_{0}, \rho\right)$, $\left[t \mapsto D_{H,(2,3,4)} \psi\left(t, x(\pi)(t), x(\pi)^{\prime}(t), \pi\right)\right]$ is measurable from $([0, T], \mathcal{B}([0, T]))$ into $\left(\left(\mathbb{R}^{n} \times \mathbb{R}^{n} \times Y\right)^{*}, \mathcal{B}\left(\left(\mathbb{R}^{n} \times \mathbb{R}^{n} \times Y\right)^{*}\right)\right.$.

Conditions on the integrands of the constraints only

(BCON) For all $\lambda=\left(\lambda_{i}\right)_{1 \leq i \leq k} \in \mathbb{R}^{k}$ and for all $\mu=\left(\mu_{j}\right)_{1 \leq j \leq \ell} \in \mathbb{R}^{\ell}$ s.t. $(\lambda, \mu) \neq$ $(0,0), x\left(\pi_{0}\right)$ is not a solution of the Euler equation in Dubois-Reymond form ([14] p.106) $D_{H, 3} \psi_{\lambda, \mu}\left(t, x(t), x^{\prime}(t), \pi_{0}\right)=\int_{[0, t]} D_{H, 2} \psi_{\lambda, \mu}\left(t, x(t), x^{\prime}(t), \pi_{0}\right) d \mathfrak{m}_{1}(t)+c_{\lambda, \mu}$ $\mathfrak{m}_{1}$-a.e. $t \in[0, T]$, where

$\psi_{\lambda, \mu}(t, x, v, \pi):=\sum_{1 \leq i \leq k} \lambda_{i} \mathfrak{g}_{i}(t, x, v, \pi)+\sum_{1 \leq j \leq \ell} \mu_{j} \mathfrak{h}_{j}(t, x, v, \pi)$ and $c_{\lambda, \mu} \in \mathbb{R}^{n *}$ is a constant.

Theorem 5.1. (Envelope Theorem). We assume that (BSOL1), (BSOL2), (BINT1), (BINT2), (BINT3) and (BCON) are fulfilled. Then the following assertions hold.

(I) The value function of $(\mathcal{V}, \pi)$ admits a right directional derivative at $\pi_{0}$ in the direction $\varpi$ exists and we have

$$
\begin{aligned}
D_{G}^{+} V\left(\pi_{0} ; \varpi\right)= & \int_{[0, T]} D_{H, 4} L\left(t, x\left(\pi_{0}\right)(t), x\left(\pi_{0}\right)^{\prime}(t), \pi_{0}\right) \cdot \varpi d \mathfrak{m}_{1}(t)+ \\
& \sum_{1 \leq i \leq k} \lambda_{i}\left(\pi_{0}\right) \int_{[0, T]} D_{H, 4} \mathfrak{g}_{i}\left(t, x\left(\pi_{0}\right)(t), x\left(\pi_{0}\right)^{\prime}(t), \pi_{0}\right) \cdot \varpi d \mathfrak{m}_{1}(t)+ \\
& \sum_{1 \leq j \leq \ell} \mu_{j}\left(\pi_{0}\right) \int_{[0, T]} D_{H, 4} \mathfrak{h}_{j}\left(t, x\left(\pi_{0}\right)(t), x\left(\pi_{0}\right)^{\prime}(t), \pi_{0}\right) \cdot \varpi d \mathfrak{m}_{1}(t)
\end{aligned}
$$

where $\left(\lambda_{i}\left(\pi_{0}\right)\right)_{1 \leq i \leq k}$ and $\left(\mu_{j}\left(\pi_{0}\right)\right)_{1 \leq j \leq \ell}$ are the Karush-Kuhn-Tucker mutipliers associated to the solution $x\left(\pi_{0}\right)$ of the problem $\left(\mathcal{V}, \pi_{0}\right)$. 
(II) If in addition we replace (BSOL2) by (BSOL2-bis), the value function is Gâteaux differentiable at $\pi_{0}$ and for all $\varpi \in Y$, we have

$$
\begin{aligned}
D_{G} V\left(\pi_{0}\right) \cdot \varpi= & \int_{[0, T]} D_{H, 4} L\left(t, x\left(\pi_{0}\right)(t), x\left(\pi_{0}\right)^{\prime}(t), \pi_{0}\right) \cdot \varpi d \mathfrak{m}_{1}(t)+ \\
& \sum_{1 \leq i \leq k} \lambda_{i}\left(\pi_{0}\right) \int_{[0, T]} D_{H, 4} \mathfrak{g}_{i}\left(t, x\left(\pi_{0}\right)(t), x\left(\pi_{0}\right)^{\prime}(t), \pi_{0}\right) \cdot \varpi d \mathfrak{m}_{1}(t)+ \\
& \sum_{1 \leq j \leq \ell} \mu_{j}\left(\pi_{0}\right) \int_{[0, T]} D_{H, 4} \mathfrak{h}_{j}\left(t, x\left(\pi_{0}\right)(t), x\left(\pi_{0}\right)^{\prime}(t), \pi_{0}\right) \cdot \varpi d \mathfrak{m}_{1}(t)
\end{aligned}
$$

(III) If in addition we assume that (BSOL2-ter), (BINT3-bis), and (BINT4) are fulfilled, then the value function is of class Fréchet $C^{1}$ on an open neighborhood of $\pi_{0}$, and for all $\pi$ which belongs to this neighborhood, for all $\varpi \in Y$, we have

$$
\begin{aligned}
D_{F} V(\pi) \cdot \varpi= & \int_{[0, T]} D_{H, 4} L\left(t, x(\pi)(t), x(\pi)^{\prime}(t), \pi\right) \cdot \varpi d \mathfrak{m}_{1}(t)+ \\
& \sum_{1 \leq i \leq k} \lambda_{i}(\pi) \int_{[0, T]} D_{H, 4} \mathfrak{g}_{i}\left(t, x(\pi)(t), x(\pi)^{\prime}(t), \pi\right) \cdot \varpi d \mathfrak{m}_{1}(t)+ \\
& \sum_{1 \leq j \leq \ell} \mu_{j}(\pi) \int_{[0, T]} D_{H, 4} \mathfrak{h}_{j}\left(t, x(\pi)(t), x(\pi)^{\prime}(t), \pi\right) \cdot \varpi d \mathfrak{m}_{1}(t)
\end{aligned}
$$

\subsection{Nonlinear Integral Functionals.}

Lemma 5.2. Let $E$ be a real normed space, $G$ be an open subset of $E, \mathfrak{f}:[0, T] \times$ $G \rightarrow \mathbb{R}$ be a function, and $z_{0} \in C^{0}([0, T], G)$. We consider the following conditions.

(i) $\mathfrak{f} \in C^{0}([0, T] \times G, \mathbb{R})$.

(ii) There exist $\rho>0$ and $\zeta \in \mathcal{L}^{1}\left([0, T], \mathcal{B}([0, T]), \mathfrak{m}_{1} ; \mathbb{R}_{+}\right)$s.t., for all $t \in[0, T]$, for all $u_{1}, u_{2} \in B\left(z_{0}(t), \rho\right),\left|\mathfrak{f}\left(t, u_{1}\right)-\mathfrak{f}\left(t, u_{2}\right)\right| \leq \zeta(t)\left\|u_{1}-u_{2}\right\|$.

(iii) For all $t \in[0, T], D_{H, 2} \mathfrak{f}\left(t, z_{0}(t)\right)$ exists.

We consider the functional $F: C^{0}([0, T], G) \rightarrow \mathbb{R}$ defined by $F(z):=\int_{0}^{T} \mathfrak{f}(t, z(t)) d t$ when $z \in C^{0}([0, T], G)$.

Then the following conclusions hold.

(a) Under (i-ii), $F$ is well defined and Lipschitzean on the ball $B_{\|\cdot\|_{\infty}}\left(z_{0}, \rho\right)$.

(b) Under (i-iii), $F$ is Hadamard differentiable at $z_{0}$, and for all

$h \in C^{0}([0, T], E),\left[t \mapsto D_{H, 2} \mathfrak{f}\left(t, z_{0}(t)\right) \cdot h(t)\right] \in \mathcal{L}^{1}\left([0, T], \mathcal{B}([0, T]), \mathfrak{m}_{1} ; \mathbb{R}\right)$ and we have $D_{H} F\left(z_{0}\right) \cdot h=\int_{[0, T]} D_{H, 2} \mathfrak{f}\left(t, z_{0}(t)\right) \cdot h(t) d \mathfrak{m}_{1}(t)$.

Proof. (a) Let $z \in C^{0}([0, T], G)$. Under (i), since $\mathfrak{f} \in C([0, T] \times G, \mathbb{R})$, we have $[t \mapsto \mathfrak{f}(t, z(t))] \in C^{0}([0, T], \mathbb{R})$; hence this function is Riemann integrable on $[0, T]$, and so $F(z)$ is well defined. Doing a straightforward majorization, we obtain the following inequality.

$$
\forall z, w \in B_{\|\cdot\|_{\infty}}\left(z_{0}, \rho\right), \quad|F(z)-F(w)| \leq\|\zeta\|_{L^{1}} \cdot\|z-w\|_{\infty} .
$$

and so the conclusion (a) is proven.

(b) When $z \in C^{0}([0, T], G)$, since $z([0, T])$ is compact and since the function $[u \mapsto d(u, E \backslash G):=\inf \{\|u-v\|: v \in E \backslash G\}]$ is continuous (since Lipschitzean), using the Optimization Theorem of Weierstrass and the closedness of $E \backslash G$, we can assert that $\alpha_{z}:=\inf \{d(u, E \backslash G): u \in z([0, T])\}>0$. We can verify that $B_{\|\cdot\|_{\infty}}\left(z, 2^{-1} \alpha_{z}\right) \subset C^{0}([0, T], G)$.

Let $h \in C^{0}([0, T], E), h \neq 0$ ( the case $h=0$ is evident).

We set $\theta^{0}:=\|h\|_{\infty}^{-1} \min \left\{\rho, 2^{-1} \alpha_{z_{0}}\right\}>0$. Hence, for all $\left.\theta \in\right] 0, \theta^{0}$, for all $t \in[0, T]$, we have $z_{0}(t)+\theta h(t) \in B\left(z_{0}(t), \rho\right)$. Let $\left.\left(\theta_{m}\right)_{m \in \mathbb{N}} \in\right] 0, \theta^{0}\left[{ }^{\mathbb{N}}\right.$ which converges to 0 . Using (iii), since the Hadamard differentiability implies the Gâteaux differentiability, we have:

$$
\left.\begin{array}{l}
D_{H, 2} \mathfrak{f}\left(t, z_{0}(t)\right) \cdot h(t)=D_{G, 2}^{+} \mathfrak{f}\left(t, z_{0}(t) ; h(t)\right) \\
=\lim _{m \rightarrow+\infty} \frac{1}{\theta_{m}}\left(\mathfrak{f}\left(t, z_{0}(t)+\theta_{m} h(t)\right)-\mathfrak{f}\left(t, z_{0}(t)\right)\right) .
\end{array}\right\}
$$


Since $\left[t \mapsto \mathfrak{f}\left(t, z_{0}(t)+\theta_{m} h(t)\right)\right]$ and $\left[t \mapsto \mathfrak{f}\left(t, z_{0}(t)\right)\right]$ belong to $C^{0}([0, T], \mathbb{R})$, they are Borel functions and therefore, for all $m \in \mathbb{N},\left[t \mapsto \frac{1}{\theta_{m}}\left(\mathfrak{f}\left(t, z_{0}(t)+\theta_{m} h(t)\right)-\mathfrak{f}\left(t, z_{0}(t)\right)\right)\right]$ is also a Borel function. Since a pointwise limit of a sequence of Borel functions is a Borel function, using (5.2), we obtain:

$$
\left[t \mapsto D_{H, 2} \mathfrak{f}\left(t, z_{0}(t)\right) \cdot h(t)\right] \in \mathcal{L}^{0}([0, T], \mathcal{B}([0, T]) ; \mathbb{R}) .
$$

Using (ii), we obtain, for all $m \in \mathbb{N}$,

$$
\left|\frac{1}{\theta_{m}}\left(\mathfrak{f}\left(t, z_{0}(t)+\theta_{m} h(t)\right)-\mathfrak{f}\left(t, z_{0}(t)\right)\right)\right| \leq \zeta(t)\|h(t)\| \leq \zeta(t)\|h\|_{\infty} .
$$

Doing $m \rightarrow+\infty$, we deduce from (5.2) and (5.4) that $\left|D_{G, 2}^{+} \mathfrak{f}\left(t, z_{0}(t) ; h(t)\right)\right| \leq$ $\zeta(t)\|h\|_{\infty}$, and consequently, for all $t \in[0, T]$, we have

$$
\left|D_{H, 2} \mathfrak{f}\left(t, z_{0}(t)\right) \cdot h(t)\right| \leq \zeta(t)\|h\|_{\infty} .
$$

From (5.3) and (5.5), we obtain the following property.

$$
\left[t \mapsto D_{H, 2} \mathfrak{f}\left(t, z_{0}(t)\right) \cdot h(t)\right] \in \mathcal{L}^{1}\left([0, T], \mathcal{B}([0, T]), \mathfrak{m}_{1} ; \mathbb{R}\right) .
$$

Note that, for all $m \in \mathbb{N}$, using (a) and the linearity of the Riemann integral, we have, for all $m \in \mathbb{N}, \frac{1}{\theta_{m}}\left(F\left(z_{0}+\theta_{m} h\right)-F\left(z_{0}\right)\right)=$ $\int_{0}^{T} \frac{1}{\theta_{m}}\left(\mathfrak{f}\left(t, z_{0}(t)+\theta_{m} h(t)\right)-\mathfrak{f}\left(t, z_{0}(t)\right)\right) d t=$ $\int_{[0, T]} \frac{1}{\theta_{m}}\left(\mathfrak{f}\left(t, z_{0}(t)+\theta_{m} h(t)\right)-\mathfrak{f}\left(t, z_{0}(t)\right)\right) d \mathfrak{m}_{1}(t)$. Then, using (5.4) and (5.2), we can use the Dominated Convergence Theorem of Lebesgue to obtain that $\lim _{n \rightarrow+\infty} \frac{1}{\theta_{m}}\left(F\left(z_{0}+\theta_{m} h\right)-F\left(z_{0}\right)\right)=\int_{[0, T]} D_{H, 2} \mathfrak{f}\left(t, z_{0}(t)\right) \cdot h(t) d \mathfrak{m}_{1}(t)$. Using the sequential characterization of the limit, we obtain the existence of $D_{G}^{+} F\left(z_{0} ; h\right)$ and

$$
D_{G}^{+} F\left(z_{0} ; h\right)=\int_{[0, T]} D_{H, 2} \mathfrak{f}\left(t, z_{0}(t)\right) \cdot h(t) d \mathfrak{m}_{1}(t) .
$$

Using the linearity of the Borel integral and the linearity of the Hadamard differential at a point, we see that $D_{G}^{+} F\left(z_{0} ; \cdot\right)$ is a linear functional from $C^{0}([0, T], E)$ into $\mathbb{R}$. Note that, using (5.7) and (5.5), we have: $\left|D_{G}^{+} F\left(z_{0} ; h\right)\right|=\mid \int_{[0, T]} D_{H, 2} \mathfrak{f}\left(t, z_{0}(t)\right)$. $h(t) d \mathfrak{m}_{1}(t)\left|\leq \int_{[0, T]}\right| D_{H, 2} \mathfrak{f}\left(t, z_{0}(t)\right) \cdot h(t) \mid d \mathfrak{m}_{1}(t) \leq \int_{[0, T]}\left(\zeta(t)\|h\|_{\infty}\right) d \mathfrak{m}_{1}(t)$ $=\|\zeta\|_{L^{1}}\|h\|_{\infty}$, and so $D_{G}^{+} F\left(z_{0} ; \cdot\right)$ is linear continuous. Hence we have proven

$$
D_{G} F\left(z_{0}\right) \text { exists. }
$$

Since $F$ is Lipschitzean, we can use $([10$, p.259) to assert that $F$ is Hadamard differentiable at $z_{0}$, and the formula of this Hadamard differential is given by this one of its Gâteaux differential.

Remark 5.3. Under the assumptions of Lemma 5.2, the following assertions hold.

(i) If $E$ is separable, then $\left[t \mapsto\left\|D_{H, 2} \mathfrak{f}\left(t, z_{0}(t)\right)\right\|_{*}\right]$ is Borel integrable on $[0, T]$.

(ii) If $E=\mathbb{R}^{n}$, then $\left[t \mapsto D_{H, 2} \mathfrak{f}\left(t, z_{0}(t)\right)\right]$ is Borel integrable on $[0, T]$

Proof. To abridge the writing, we set $\Lambda(t):=D_{H, 2} \mathfrak{f}\left(t, z_{0}(t)\right)$.

(i) Since $E$ is separable (and metric), the closed unit ball of $E, \bar{B}_{E}(0,1)$, is also separable $\left([9],(3.10 .9)\right.$, p.45), hence there exists $A \subset \bar{B}_{E}(0,1)$ which is at most countable and dense in $\bar{B}_{E}(0,1)$. Using (5.6), for all $t \in[0, T],\|\Lambda(t)\|_{*}=$ $\sup \left\{|\Lambda(t) \cdot v|: v \in \bar{B}_{E}(0,1)\right\}=\sup \{|\Lambda(t) \cdot v|: v \in A\} \in \mathcal{L}^{0}([0, T], \mathcal{B}([0, T]) ; \mathbb{R})$ as a supremum of a sequence of functions which belong to $\mathcal{L}^{0}([0, T], \mathcal{B}([0, T]) ; \mathbb{R})^{\mathbb{N}}$. Using (5.5) we obtain that, for all $t \in[0, T],\|\Lambda(t)\|_{*} \leq \zeta(t)$, which implies that 
$\|\Lambda(\cdot)\|_{*} \in \mathcal{L}^{1}\left([0, T], \mathcal{B}([0, T]), \mathfrak{m}_{1} ; \mathbb{R}_{+}\right)$.

(ii) Since $\operatorname{dim} \mathbb{R}^{n *}$ is finite, the $\mathfrak{m}_{1}$-integrability of $\Lambda$ is equivalent to the $\mathfrak{m}_{1}$ integrability of its coordinate functions. Let $\left(e_{i}\right)_{1 \leq i \leq n}$ be the canonical basis of $\mathbb{R}^{n}$ and $\left(e_{i}^{*}\right)_{1 \leq i \leq n}$ its dual basis. Note that we have $\Lambda(t)=\sum_{1 \leq i \leq n}\left(\Lambda(t) \cdot e_{i}\right) e_{i}^{*}$. From (5.6) we know that, for all $i \in\{1, \ldots, n\}, \Lambda(\cdot) \cdot e_{i}$ is $\mathfrak{m}_{1}$-integrable on $[0, T]$, and consequently we obtain that $\Lambda \in \mathcal{L}^{1}\left([0, T], \mathcal{B}([0, T]), \mathfrak{m}_{1} ; \mathbb{R}^{n *}\right)$.

Remark 5.4. Consider the following strengthened condition:

(St) $: \mathfrak{f} \in C^{0}([0, T] \times G, \mathbb{R}), D_{F, 2} \mathfrak{f}(t, \cdot)$ exists on $G$ for all $t \in[0, T]$, and $D_{F, 2} \mathfrak{f} \in$ $C^{0}\left([0, T] \times G, E^{*}\right)$.

$\operatorname{Under}(S t)$, the Nemytskii operator $\left.\mathcal{N}_{\mathfrak{f}}: C^{0}([0, T], G)\right) \rightarrow C^{0}([0, T], \mathbb{R})$, defined by $\mathcal{N}_{\mathfrak{f}}(z)(t):=\mathfrak{f}(t, z(t))$ for all $t \in[0, T]$ and for all $z \in C^{0}([0, T], G)$, is Fréchet $C^{1}$ and $\left(D_{F} \mathcal{N}_{\mathfrak{f}}(z) \cdot h\right)(t)=D_{F, 2} \mathfrak{f}(t, z(t)) \cdot h(t)$ for all $t \in[0, T]$ and for all $h \in$ $C^{0}([0, T], E)$. This result is proven in $[3]$ (Lemma 12). Since the Riemann integral defines a linear continuous functional $\mathcal{I}$ on $C^{0}([0, T], \mathbb{R})$, the functional $F$, defined by $F(z):=\int_{0}^{T} \mathfrak{f}(t, z(t)) d t$, verifies $F=\mathcal{I} \circ \mathcal{N}_{\mathfrak{f}}$, and therefore $F$ is Fréchet $C^{1}$, and, using the Chain Rule, we have $D_{F} F(z) \cdot h=\int_{0}^{T} D_{F, 2} \mathfrak{f}(t, z(t)) \cdot h(t) d t$. Under (St), the assumptions (i) and (iii) of Lemma 5.2 are fulfilled. Using Lemma 12 of [3], the Nemytskii operator $\mathcal{N}_{D_{F, 2} f}$ is continuous, and since a mapping which is continuous at a point is bounded on a neighborhood of this point, and using the Mean Value Inequality we see that (ii) of Lemma 5.2 is fulfilled. Hence Lemma 5.2 contains a contribution to improve the results of [3] on the differentiability of the nonlinear integral functionals.

Lemma 5.5. let $M$ be an open subset of $\mathbb{R}^{n}, \mathbf{P}$ be an open subset of $Y, \phi$ : $[0, T] \times M \times \mathbb{R}^{n} \times \mathbf{P} \rightarrow \mathbb{R}$ be a function, $\pi_{0} \in \mathbf{P}$ and $[\pi \mapsto \mathbf{x}(\pi)]$ be a mapping from $\mathbf{P}$ into $C^{1}([0, T], M)$. We consider the following conditions.

(i) $\phi \in C^{0}\left([0, T] \times M \times \mathbb{R}^{n} \times \mathbf{P}, \mathbb{R}\right)$.

(ii) $[\pi \mapsto \mathbf{x}(\pi)]$ is continuous at $\pi_{0}$.

(iii) There exist $\varrho>0$ and $\gamma \in \mathcal{L}^{1}\left([0, T], \mathcal{B}([0, T]), \mathfrak{m}_{1} ; \mathbb{R}_{+}\right)$s.t. $\forall t \in[0, T]$, $\forall u, u_{1} \in B\left(\mathbf{x}\left(\pi_{0}\right)(t), \varrho\right), \forall v, v_{1} \in B\left(\mathbf{x}\left(\pi_{0}\right)^{\prime}(t), \varrho\right), \forall \pi, \pi_{1} \in B\left(\pi_{0}, \varrho\right)$, $\left|\phi(t, u, v, \pi)-\phi\left(t, u_{1}, v_{1}, \pi_{1}\right)\right| \leq \gamma(t)\left(\left\|u-u_{1}\right\|+\left\|v-v_{1}\right\|+\left\|\pi-\pi_{1}\right\|\right)$.

(iv) For all $t \in[0, T]$, for all $\pi \in B\left(\pi_{0}, \varrho\right), D_{H,(2,3,4)} \phi\left(t, \mathbf{x}(\pi)(t), \mathbf{x}(\pi)^{\prime}(t), \pi\right)$ exists.

(v) For all $t \in[0, T],\left[\pi \mapsto D_{H,(2,3)} \phi\left(t, \mathbf{x}(\pi)(t), \mathbf{x}(\pi)^{\prime}(t), \pi\right)\right]$ is continuous on $B\left(\pi_{0}, \varrho\right)$.

(vi) For all $t \in[0, T],\left[\pi \mapsto D_{H,(2,3,4)} \phi\left(t, \mathbf{x}(\pi)(t), \mathbf{x}(\pi)^{\prime}(t), \pi\right)\right]$ is continuous on $B\left(\pi_{0}, \varrho\right)$.

(vii) For all $\pi \in B\left(\pi_{0}, \varrho\right),\left[t \mapsto D_{H,(2,3,4)} \phi\left(t, \mathbf{x}(\pi)(t), \mathbf{x}(\pi)^{\prime}(t), \pi\right)\right]$ belongs to $\mathcal{L}^{0}\left([0, T], \mathcal{B}([0, T]) ;\left(\mathbb{R}^{n} \times \mathbb{R}^{n} \times Y\right)^{*}\right)$.

We consider the functional $\Phi: C^{1}([0, T], M) \times \mathbf{P} \rightarrow \mathbb{R}$ defined by $\Phi(\mathbf{x}, \pi):=$ $\int_{0}^{T} \phi\left(t, \mathbf{x}(t), \mathbf{x}^{\prime}(t), \pi\right) d t$. Then the following conclusions hold.

(a) Under (i-iii), there exists $\sigma \in] 0, \varrho]$ s.t. for all $\pi \in B\left(\pi_{0}, \sigma\right),[\mathbf{x} \mapsto \Phi(\mathbf{x}, \pi)]$ is Lipschitzean on $B_{C^{1}}(\mathbf{x}(\pi), \sigma)$.

(b) Under (i-iv), for all $\pi \in B\left(\pi_{0}, \sigma\right), D_{H} \Phi(\mathbf{x}(\pi), \pi)$ exists, and for all $\mathbf{h} \in$ $C^{1}\left([0, T], \mathbb{R}^{n}\right)$, for all $\varpi \in Y$, $\left[t \mapsto D_{H,(2,3,4)} \phi\left(t, \mathbf{x}(\pi)(t), \mathbf{x}(\pi)^{\prime}(t), \pi\right) \cdot\left(\mathbf{h}(t), \mathbf{h}^{\prime}(t), \varpi\right)\right]$ belongs to 


$$
\begin{aligned}
& \mathcal{L}^{1}\left([0, T], \mathcal{B}([0, T]), \mathfrak{m}_{1} ; \mathbb{R}\right) \text { and } \\
& D_{H} \Phi(\mathbf{x}(\pi), \pi) \cdot(\mathbf{h}, \varpi)= \int_{[0, T]} D_{H, 2} \phi\left(t, \mathbf{x}(\pi)(t), \mathbf{x}(\pi)^{\prime}(t), \pi\right) \cdot \mathbf{h}(t) d \mathfrak{m}_{1}(t) \\
&+\int_{[0, T]} D_{H, 3} \phi\left(t, \mathbf{x}(\pi)(t), \mathbf{x}(\pi)^{\prime}(t), \pi\right) \cdot \mathbf{h}^{\prime}(t) d \mathfrak{m}_{1}(t) \\
&+\int_{[0, T]} D_{H, 4} \phi\left(t, \mathbf{x}(\pi)(t), \mathbf{x}(\pi)^{\prime}(t), \pi\right) \cdot \varpi d \mathfrak{m}_{1}(t) .
\end{aligned}
$$

(c) Under (i-v), $\left[\pi \mapsto D_{H, 1} \Phi(\mathbf{x}(\pi), \pi)\right]$ is continuous from $B\left(\pi_{0}, \sigma\right)$ into $\left(C^{1}\left([0, T], \mathbb{R}^{n}\right)\right)^{*}$.

(d) Under (i-vii), $\left[\pi \mapsto D_{H} \Phi(\mathbf{x}(\pi), \pi)\right]$ is continuous from $B\left(\pi_{0}, \sigma\right)$ into $\left(C^{1}\left([0, T], \mathbb{R}^{n}\right) \times Y\right)^{*}$.

Proof. Let $\varrho$ be given by (iii). Using (ii), there exists $\left.\sigma \in] 0, \frac{\varrho}{2}\right]$ s.t. $\| \mathbf{x}(\pi)-$ $\mathbf{x}\left(\pi_{0}\right) \|_{C^{1}} \leq \frac{\varrho}{2}$ when $\left\|\pi-\pi_{0}\right\|<\sigma$. Using (iii), we obtain the following property.

$$
\begin{aligned}
& \forall \pi \in B\left(\pi_{0}, \sigma\right), \forall t \in[0, T], \forall u_{1}, u_{2} \in B(\mathbf{x}(\pi)(t), \sigma), \forall v_{1}, v_{2} \in B\left(\mathbf{x}(\pi)^{\prime}(t), \sigma\right), \\
& \forall \pi_{1}, \pi_{2} \in B(\pi, \sigma),\left|\phi\left(t, u_{1}, v_{1}, \pi_{1}\right)-\phi\left(t, u_{2}, v_{2}, \pi_{2}\right)\right| \leq \\
& \gamma(t)\left(\left\|u_{1}-u_{2}\right\|+\left\|v_{1}-v_{2}\right\|+\left\|\pi_{1}-\pi_{2}\right\|\right)
\end{aligned}
$$

We want to use Lemma 5.2, We set $E:=\mathbb{R}^{n} \times \mathbb{R}^{n} \times Y$ and $G:=M \times \mathbb{R}^{n} \times \mathbf{P}$ which is an open subset of $E$. At each $\pi \in \mathbf{P}$ we associate the constant mapping $\pi_{c}:=[t \mapsto \pi] \in C^{0}([0, T], \mathbf{P})$. We define the function $\mathfrak{f}:[0, T] \times G \rightarrow \mathbb{R}$ by setting $\mathfrak{f}(t,(u, v, \pi)):=\phi(t, u, v, \pi)$. When $\mathbf{x} \in C^{1}([0, T], M)$ and $\pi \in \mathbf{P}$, setting $z(t):=\left(\mathbf{x}(t), \mathbf{x}^{\prime}(t), \pi_{c}(t)\right)$, we have $z \in C^{0}([0, T], G)$, and $F(z)=\int_{0}^{T} \mathfrak{f}(t, z(t)) d t=$ $\int_{0}^{T} \phi\left(t, \mathbf{x}(t), \mathbf{x}^{\prime}(t), \pi\right) d t=\Phi(\mathbf{x}, \pi)$. Hence $\Phi$ can be viewed as a restriction of $F$ to an open subset of a closed (since complete) vector subspace.

Proof of (a). Let $\pi \in B\left(\pi_{0}, \sigma\right)$; we set $z_{0}(t):=\left(\mathbf{x}(\pi)(t), \mathbf{x}(\pi)^{\prime}(t),(\pi)_{c}(t)\right)$. Now we verify that that the assumptions of Lemma 5.2 are fulfilled. Note that (i) implies that the assumption (i) of Lemma 5.2 is fulfilled. After (5.9), the assumption (ii) of Lemma 5.2 is fulfilled. Hence we can use the conclusion (a) of Lemma 5.2 to ensure that $[\mathbf{x} \mapsto \Phi(\mathbf{x}, \pi)]$ is Lipschitzean on $B_{C^{1}}(\mathbf{x}(\pi), \sigma)$, and so the conclusion (a) is proven.

Proof of (b). We arbitrarily fix $\pi \in B\left(\pi_{0}, \sigma\right)$, and we set $z_{0}(t):=\left(\mathbf{x}(\pi)(t), \mathbf{x}(\pi)^{\prime}(t), \pi_{c}(t)\right)$. We can verify that assumptions of Lemma 5.2 are fulfilled for $z_{0}$. In the proof of (a) we have yet proved that assumptions (i) and (ii) of Lemma 5.2 are fulfilled. Moreover assumption (iv) of Lemma [5.5] implies that the assumption (iii) of Lemma 5.2 is fulfilled. Hence we can use the conclusion (b) of Lemma 5.2 and assert that $D_{H} \Phi(\mathbf{x}(\pi), \pi)=D_{H} F\left(z_{0}\right)$ exists. We introduce the operator $\Psi: C^{1}\left([0, T], \mathbb{R}^{n}\right) \times Y \rightarrow C^{0}\left([0, T], \mathbb{R}^{n}\right) \times C^{0}\left([0, T], \mathbb{R}^{n}\right) \times Y$ defined by $\Psi(\mathbf{x}, \pi):=\left(\mathbf{x}, \mathbf{x}^{\prime}, \pi\right)$. $\Psi$ is linear continuous, hence it is Fréchet differentiable, and consequently Hadamard differentiable. We set $\Psi_{0}$ the restriction of $\Psi$ to $C^{1}([0, T], M) \times \mathbf{P}$ and we note that $\Phi=F \circ \Psi_{0}$. Using Lemma [5.2, we know that $\left[t \mapsto D_{H, 2} \mathfrak{f}\left(t, z_{0}(t)\right) \cdot w(t)\right]$ belongs to $\mathcal{L}^{1}\left([0, T], \mathcal{B}([0, T]), \mathfrak{m}_{1} ; \mathbb{R}\right)$ for all $w \in C^{0}([0, T], E)$, and $D_{H} F\left(z_{0}\right) \cdot w=\int_{[0, T]} D_{H, 2} \mathfrak{f}\left(t, z_{0}(t)\right) \cdot w(t) d \mathfrak{m}_{1}(t)$. Note that $D_{H, 2} \mathfrak{f}\left(t, z_{0}(t)\right)=D_{H,(2,3,4)} \phi\left(t, \mathbf{x}(\pi)(t), \mathbf{x}(\pi)^{\prime}(t), \pi\right)$ and $D_{H} \Psi_{0}(\mathbf{x}(\pi), \pi) \cdot(\mathbf{h}, \varpi)=$ $\Psi(\mathbf{h}, \varpi)=\left(\mathbf{h}, \mathbf{h}^{\prime}, \varpi\right)$, and using the Chain Rule, we obtain the following formula.

$$
\left.\begin{array}{l}
D_{H} \Phi(\mathbf{x}(\pi), \pi) \cdot(\mathbf{h}, \varpi)= \\
\int_{[0, T]} D_{H,(2,3,4)} \phi\left(t, \mathbf{x}(\pi)(t), \mathbf{x}(\pi)^{\prime}(t), \pi\right) \cdot\left(\mathbf{h}(t), \mathbf{h}^{\prime}(t), \varpi\right) d \mathfrak{m}_{1}(t) .
\end{array}\right\}
$$


From this last relation we deduce the formula of the conclusion (b).

Proof of (c). From (5.10) we deduce the following formula.

$$
\left.\begin{array}{l}
D_{H, 1} \Phi(\mathbf{x}(\pi), \pi) \cdot \mathbf{h}= \\
\int_{[0, T]} D_{H,(2,3)} \phi\left(t, \mathbf{x}(\pi)(t), \mathbf{x}(\pi)^{\prime}(t), \pi\right) \cdot\left(\mathbf{h}(t), \mathbf{h}^{\prime}(t)\right) d \mathfrak{m}_{1}(t) .
\end{array}\right\}
$$

Let $\pi \in B\left(\pi_{0}, \sigma\right)$ and $\left(\pi_{k}\right)_{k \in \mathbb{N}} \in B\left(\pi_{0}, \sigma\right)^{\mathbb{N}}$ which converges to $\pi$. When $k \in \mathbb{N}$, and $t \in[0, T]$, we set

$$
\Gamma_{k}(t):=\left\|D_{H,(2,3)} \phi\left(t, \mathbf{x}\left(\pi_{k}\right)(t), \mathbf{x}\left(\pi_{k}\right)^{\prime}(t), \pi_{k}\right)-D_{H,(2,3)} \phi\left(t, \mathbf{x}(\pi)(t), \mathbf{x}(\pi)^{\prime}(t), \pi\right)\right\|_{*}
$$

where the norm is the norm of $\left(\mathbb{R}^{n} \times \mathbb{R}^{n}\right)^{*}$. From (5.9) and (v), we obtain the following properties.

$$
\forall t \in[0, T], \Gamma_{k}(t) \leq 2 \gamma(t), \text { and } \lim _{k \rightarrow+\infty} \Gamma_{k}(t)=0 .
$$

Let $p \in\{\pi\} \cup\left\{\pi_{k}: k \in \mathbb{N}\right\}$. Since, for all $\mathbf{h}, \mathbf{k} \in C^{0}\left([0, T], \mathbb{R}^{n}\right)$, $\left[t \mapsto D_{H,(2,3)} \phi\left(t, \mathbf{x}(p)(t), \mathbf{x}(p)^{\prime}(t), p\right) \cdot(\mathbf{h}(t), \mathbf{k}(t))\right] \in \mathcal{L}^{1}\left([0, T], \mathcal{B}([0, T]), \mathfrak{m}_{1} ; \mathbb{R}\right)$, we have that $\left[t \mapsto D_{H,(2,3)} \phi\left(t, \mathbf{x}(p)(t), \mathbf{x}(p)^{\prime}(t), p\right) \cdot(\mathbf{h}(t), \mathbf{k}(t))\right] \in \mathcal{L}^{0}([0, T], \mathcal{B}([0, T]) ; \mathbb{R})$. Hence, for all $v, w \in \mathbb{R}^{n}$,

$\left[t \mapsto D_{H,(2,3)} \phi\left(t, \mathbf{x}(p)(t), \mathbf{x}(p)^{\prime}(t), p\right) \cdot(v, w)\right] \in \mathcal{L}^{0}([0, T], \mathcal{B}([0, T]) ; \mathbb{R})$. Let $\left(e_{i}\right)_{1 \leq i \leq 2 n}$ be the canonical basis of $\mathbb{R}^{n} \times \mathbb{R}^{n}$, and $\left(e_{i}^{*}\right)_{1 \leq i \leq 2 n}$ denotes its dual basis. Note that, for all $t \in[0, T]$, we have

$$
D_{H,(2,3)} \phi\left(t, \mathbf{x}(p)(t), \mathbf{x}(p)^{\prime}(t), p\right)=\sum_{1 \leq i \leq 2 n}\left(D_{H,(2,3)} \phi\left(t, \mathbf{x}(p)(t), \mathbf{x}(p)^{\prime}(t), p\right) \cdot e_{i}\right) e_{i}^{*},
$$

hence, as a composition of Borel functions, we obtain

$$
\left[t \mapsto D_{H,(2,3)} \phi\left(t, \mathbf{x}(p)(t), \mathbf{x}(p)^{\prime}(t), p\right)\right] \in \mathcal{L}^{0}\left([0, T], \mathcal{B}([0, T]) ;\left(\mathbb{R}^{n} \times \mathbb{R}^{n}\right)^{*}\right) .
$$

As compositions of Borel functions, we deduce of this property that

$$
\forall k \in \mathbb{N},\left[t \mapsto \Gamma_{k}(t)\right] \in \mathcal{L}^{0}([0, T], \mathcal{B}([0, T]) ; \mathbb{R}) .
$$

Since $\gamma$ is $\mathfrak{m}_{1}$-integrable on $[0, T]$, from (5.13) and (5.12), we obtain that $\Gamma_{k}$ is $\mathfrak{m}_{1^{-}}$ integrable on $[0, T]$ for all $k \in \mathbb{N}$. Hence we can do the following majorizations. For all $k \in \mathbb{N}$, for all $\mathbf{h} \in C^{1}\left([0, T], \mathbb{R}^{n}\right)$ s.t. $\|\mathbf{h}\|_{C^{1}} \leq 1$, we have

$$
\begin{aligned}
& \left.\mid D_{H, 1} \Phi\left(\mathbf{x}\left(\pi_{k}\right), \pi_{k}\right) \cdot h-D_{H, 1} \Phi(\mathbf{x}(\pi)), \pi\right) \cdot \mathbf{h} \mid \\
& =\mid \int_{[0, T]}\left(D_{H,(2,3)} \phi\left(t, \mathbf{x}\left(\pi_{k}\right)(t), \mathbf{x}\left(\pi_{k}\right)^{\prime}(t), \pi_{k}\right)\right. \\
& \left.-D_{H,(2,3)} \phi\left(t, \mathbf{x}(\pi)(t), \mathbf{x}(\pi)^{\prime}(t), \pi\right)\right) \cdot\left(\mathbf{h}(t), \mathbf{h}^{\prime}(t)\right) d \mathfrak{m}_{1}(t) \mid \\
& \leq \int_{[0, T]}\left(\Gamma_{k}(t)\left\|\left(\mathbf{h}(t), \mathbf{h}^{\prime}(t)\right)\right\| d \mathfrak{m}_{1}(t) \leq\left(\int_{[0, T]} \Gamma_{k}(t) d \mathfrak{m}_{1}(t)\right)\|\mathbf{h}\|_{C^{1}} .\right.
\end{aligned}
$$

Taking the supremum on the $\mathbf{h} \in C^{1}\left([0, T], \mathbb{R}^{n}\right)$ s.t. $\|\mathbf{h}\|_{C^{1}} \leq 1$, we obtain

$$
\left.\| D_{H, 1} \Phi\left(\mathbf{x}\left(\pi_{k}\right), \pi_{k}\right)-D_{H, 1} \Phi(\mathbf{x}(\pi)), \pi\right) \|_{\left(C^{1}\left([0, T], \mathbb{R}^{n}\right)\right)^{*}} \leq \int_{[0, T]} \Gamma_{k}(t) d \mathfrak{m}_{1}(t) .
$$

Using (5.14) and (5.12) we can apply the dominated convergence theorem of Lebesgue to obtain that

$\left.\lim _{k \rightarrow+\infty} \| D_{H, 1} \Phi\left(\mathbf{x}\left(\pi_{k}\right), \pi_{k}\right)-D_{H, 1} \Phi(\mathbf{x}(\pi)), \pi\right) \|_{\left(C^{1}\left([0, T], \mathbb{R}^{n}\right)\right)^{*}}=0$, and using the sequential characterization of the continuity, we have proven the conclusion (c).

Proof of (d). Let $\pi \in B\left(\pi_{0}, \sigma\right)$ and $\left(\pi_{k}\right)_{k \in \mathbb{N}} \in B\left(\pi_{0}, \sigma\right)^{\mathbb{N}}$ which converges to $\pi$. When $k \in \mathbb{N}$ and $t \in[0, T]$, we set

$\Delta_{k}(t):=\left\|D_{H,(2,3,4)} \phi\left(t, \mathbf{x}\left(\pi_{k}\right)(t), \mathbf{x}\left(\pi_{k}\right)^{\prime}(t), \pi_{k}\right)-D_{H,(2,3,4)} \phi\left(t, \mathbf{x}(\pi)(t), \mathbf{x}(\pi)^{\prime}(t), \pi\right)\right\|_{*}$, 
where the norm is the norm of $\left(\mathbb{R}^{n} \times \mathbb{R}^{n} \times Y\right)^{*}$. Using (vii), as compositions of Borel functions we have

$$
\forall k \in \mathbb{N},\left[t \mapsto \Delta_{k}(t)\right] \in \mathcal{L}^{0}\left([0, T], \mathcal{B}([0, T]) ; \mathbb{R}_{+}\right) .
$$

Proceeding as in the proof of (c) to establish (5.14), we obtain, for all $\varpi \in Y$ and for all $\mathbf{h} \in C^{1}\left([0, T], \mathbb{R}^{n}\right)$ s.t. $\|\mathbf{h}\|_{C^{1}}+\|\varpi\| \leq 1$,

$\left.\mid D_{H} \Phi\left(\mathbf{x}\left(\pi_{k}\right), \pi_{k}\right) \cdot(\mathbf{h}, \varpi)-D_{H} \Phi(\mathbf{x}(\pi)), \pi\right) \cdot(\mathbf{h}, \varpi) \mid \leq\left(\int_{[0, T]} \Delta_{k}(t) d \mathfrak{m}_{1}(t)\right)\left(\|\mathbf{h}\|_{C^{1}}+\right.$ $\|\varpi\|)$, and taking the l.u.b. on the $(\mathbf{h}, \varpi)$ s.t. $\|\mathbf{h}\|_{C^{1}}+\|\varpi\| \leq 1$, we obtain, for all $k \in \mathbb{N}$,

$$
\left\|D_{H} \Phi\left(\mathbf{x}\left(\pi_{k}\right), \pi_{k}\right)-D_{H} \Phi(\mathbf{x}(\pi), \pi)\right\|_{\left(C^{1}\left([0, T], \mathbb{R}^{n}\right) \times Y\right)^{*}} \leq \int_{[0, T]} \Delta_{k}(t) d \mathfrak{m}_{1}(t) .
$$

From assumption (vi), we deduce that $\lim _{k \rightarrow+\infty} \Delta_{k}(t)=0$ for all $t \in[0, T]$. Proceeding as in the proof of (c), we obtain $0 \leq \Delta_{k}(t) \leq 2 \gamma(t)$ for all $k \in \mathbb{N}$ and for all $t \in[0, T]$. Then we can use the dominated convergence theorem of Lebesgue to obtain $\lim _{k \rightarrow+\infty} \int_{[0, T]} \Delta_{k}(t) d \mathfrak{m}_{1}(t)=0$. From (5.14), we deduce that $\lim _{k \rightarrow+\infty}\left\|D_{H} \Phi\left(\mathbf{x}\left(\pi_{k}\right), \pi_{k}\right)-D_{H} \Phi(\mathbf{x}(\pi), \pi)\right\|_{\left(C^{1}\left([0, T], \mathbb{R}^{n}\right) \times Y\right)^{*}}=0$, and using the sequential characterization of the continuity, (d) is proven.

Remark 5.6. Working as in the proof of Remark 5.3, if $Y$ is separable, under the assumption (vii) we obtain that $\left[t \mapsto \Delta_{k}(t)\right]$ is a Borel function without to use assumption (vii).

Remark 5.7. We fix $\pi \in B\left(\pi_{0}, \sigma\right)$. The property on the integrability of $[t \mapsto$ $\left.D_{H,(2,3,4)} \phi\left(t, \mathbf{x}(\pi)(t), \mathbf{x}(\pi)^{\prime}(t), \pi\right) \cdot\left(\mathbf{h}(t), \mathbf{h}^{\prime}(t), \varpi\right)\right]$ given in the conclusion (b) of Lemma 5.5 implies the two following properties.

$$
\begin{aligned}
& {\left[t \mapsto D_{H, 2} \phi\left(t, \mathbf{x}(\pi)(t), \mathbf{x}(\pi)^{\prime}(t), \pi\right)\right] \in \mathcal{L}^{1}\left([0, T], \mathcal{B}([0, T]), \mathbb{R}^{n *} ; \mathfrak{m}_{1}\right) .} \\
& {\left[t \mapsto D_{H, 3} \phi\left(t, \mathbf{x}(\pi)(t), \mathbf{x}(\pi)^{\prime}(t), \pi\right)\right] \in \mathcal{L}^{1}\left([0, T], \mathcal{B}([0, T]), \mathbb{R}^{n *} ; \mathfrak{m}_{1}\right) .}
\end{aligned}
$$

To prove them we consider $\left(e_{i}\right)_{1 \leq i \leq n}$ the canonical basis of $\mathbb{R}^{n}$; we denote by $\mathbf{e}_{i}$ the constant function on $[0, T]$ equal to $e_{i}$. We have $\mathbf{e}_{i} \in C^{1}\left([0, T], \mathbb{R}^{n}\right)$ and $\mathbf{e}_{i}^{\prime}=0$. Hence the function $\left[t \mapsto D_{H, 2} \phi\left(t, \mathbf{x}(\pi)(t), \mathbf{x}(\pi)^{\prime}(t), \pi\right) \cdot e_{i}=\right.$ $\left.D_{H,(2,3,4)} \phi\left(t, \mathbf{x}(\pi)(t), \mathbf{x}(\pi)^{\prime}(t), \pi\right) \cdot\left(\mathbf{e}_{i}(t), 0,0\right)\right]$ is $\mathfrak{m}_{1}$-integrable on $[0, T]$ for all $i \in$ $\{1, \ldots, n\}$, and so (b1) is proven.

Now we consider the function $\mathbf{a}_{i}:=\left[t \mapsto t e_{i}\right] \in C^{1}\left([0, T], \mathbb{R}^{n}\right)$ for all $i \in\{1, \ldots, n\}$. Hence the function $\left[t \mapsto D_{H, 2} \phi\left(t, \mathbf{x}(\pi)(t), \mathbf{x}(\pi)^{\prime}(t), \pi\right) \cdot \mathbf{a}_{i}(t)+\right.$

$\left.\left.D_{H, 3} \phi\left(t, \mathbf{x}(\pi)(t), \mathbf{x}(\pi)^{\prime}(t), \pi\right) \cdot e_{i}\right)=D_{H,(2,3,4)} \phi\left(t, \mathbf{x}(\pi)(t), \mathbf{x}(\pi)^{\prime}(t), \pi\right) \cdot\left(\mathbf{a}_{i}(t), \mathbf{a}_{i}^{\prime}(t), 0\right)\right]$ is $\mathfrak{m}_{1}$-integrable on $[0, T]$ for all $i \in\{1, \ldots, n\}$.

Note that $\left[t \mapsto D_{H, 2} \phi\left(t, \mathbf{x}(\pi)(t), \mathbf{x}(\pi)^{\prime}(t), \pi\right) \cdot\left(t_{i}\right)\right]$ is a Borel function, and we have, for all $t \in[0, T]$,

$\left|D_{H, 2} \phi\left(t, \mathbf{x}(\pi)(t), \mathbf{x}(\pi)^{\prime}(t), \pi\right) \cdot\left(t e_{i}\right)\right| \leq\left\|D_{H, 2} \phi\left(t, \mathbf{x}(\pi)(t), \mathbf{x}(\pi)^{\prime}(t), \pi\right)\right\| T\left\|e_{i}\right\|$ which is $\mathfrak{m}_{1}$-integrable on $[0, T]$. Therefore $\left[t \mapsto D_{H, 2} \phi\left(t, \mathbf{x}(\pi)(t), \mathbf{x}(\pi)^{\prime}(t), \pi\right) \cdot\left(t e_{i}\right)\right]$ is $\mathfrak{m}_{1}$-integrable on $[0, T]$. Since $D_{H, 3} \phi\left(t, \mathbf{x}(\pi)(t), \mathbf{x}(\pi)^{\prime}(t), \pi\right) \cdot e_{i}=$ $\left[D_{H, 2} \phi\left(t, \mathbf{x}(\pi)(t), \mathbf{x}(\pi)^{\prime}(t), \pi\right) \cdot\left(t e_{i}\right)\right.$ $\left.+D_{H, 3} \phi\left(t, \mathbf{x}(\pi)(t), \mathbf{x}(\pi)^{\prime}(t), \pi\right) \cdot e_{i}-D_{H, 2} \phi\left(t, \mathbf{x}(\pi)(t), \mathbf{x}(\pi)^{\prime}(t), \pi\right) \cdot\left(t e_{i}\right)\right]$, we obtain that $\left[t \mapsto D_{H, 3} \phi\left(t, \mathbf{x}(\pi)(t), \mathbf{x}(\pi)^{\prime}(t), \pi\right) \cdot e_{i}\right]$ is $\mathfrak{m}_{1}$-integrable on $[0, T]$ as a difference of $\mathfrak{m}_{1}$-integrable functions for all $i \in\{1, \ldots, n\}$; and so (b2) is proven. 


\subsection{Euler equation.}

Lemma 5.8. In the setting of Lemma 5.5, let $\pi \in B\left(\pi_{0}, \alpha\right)$. Under conditions (i-iv) of Lemma 5.5. The two following assertions are equivalent.

(i) $\forall \mathbf{h} \in C_{0,0}^{1}\left([0, T], \mathbb{R}^{n}\right), \quad D_{H, 1} \Phi(\mathbf{x}(\pi), \pi) \cdot \mathbf{h}=0$.

(ii) There exists $c \in \mathbb{R}^{n *}$ s.t. $\mathfrak{m}_{1}$-a.e. $t \in[0, T]$,

$D_{H, 3} \phi\left(t, \mathbf{x}(\pi)(t), \mathbf{x}(\pi)^{\prime}(t), \pi\right)=\int_{[0, t]} D_{H, 2} \phi\left(s, \mathbf{x}(\pi)(s), \mathbf{x}(\pi)^{\prime}(s), \pi\right) d \mathfrak{m}_{1}(s)+$ c.

Proof. Setting $\mathfrak{M}(t):=D_{H, 2} \phi\left(t, \mathbf{x}(\pi)(t), \mathbf{x}(\pi)^{\prime}(t), \pi\right)$ and

$\mathfrak{N}(t):=D_{H, 3} \phi\left(t, \mathbf{x}(\pi)(t), \mathbf{x}(\pi)^{\prime}(t), \pi\right)$ when $t \in[0, T]$, using Lemma 5.5. we obtain that, for all $\mathbf{h} \in C_{c}^{\infty}\left([0, T], \mathbb{R}^{n}\right)$,

$$
D_{H, 1} \Phi(\mathbf{x}(\pi), \pi) \cdot h=\int_{[0, T]}\left(\mathfrak{M}(t) \cdot \mathbf{h}(t)+\mathfrak{N}(t) \cdot \mathbf{h}^{\prime}(t)\right) d \mathfrak{m}_{1}(t) .
$$

From Remark [5.7, we know that $\mathfrak{M}, \mathfrak{N} \in \mathcal{L}^{1}\left([0, T], \mathcal{B}([0, T]), \mathfrak{m}_{1} ; \mathbb{R}^{n *}\right)$. We define $\mathfrak{P}(t):=\int_{[0, t]} \mathfrak{M}(s) d \mathfrak{m}_{1}(s)$; we have $\mathfrak{P} \in A C\left([0, T], \mathbb{R}^{n *}\right)$ and $\mathfrak{P}^{\prime}(t)=\mathfrak{M}(t) \quad \mathfrak{m}_{1}$-a.e. $t \in[0, T]$. When $\mathbf{h} \in C_{c}^{\infty}\left([0, T], \mathbb{R}^{n}\right), \mathfrak{P} \cdot \mathbf{h} \in A C([0, T], \mathbb{R})$ and the formula of integration by parts $\left([13]\right.$, Annexe) holds: $\int_{[0, T]} \mathfrak{P}^{\prime}(t) \cdot \mathbf{h}(t) d \mathfrak{m}_{1}(t)=\mathfrak{P}(T) \cdot \mathbf{h}(T)-$ $\mathfrak{P}(0) \cdot \mathbf{h}(0)-\int_{[0, T]} \mathfrak{P}(t) \cdot \mathbf{h}^{\prime}(t) d \mathfrak{m}_{1}(t)$, and since $\mathbf{h}(T)=\mathbf{h}(0)=0$, we have

$$
\int_{[0, T]} \mathfrak{P}^{\prime}(t) \cdot \mathbf{h}(t) d \mathfrak{m}_{1}(t)=-\int_{[0, T]} \mathfrak{P}(t) \cdot \mathbf{h}^{\prime}(t) d \mathfrak{m}_{1}(t) .
$$

$[(\mathbf{i}) \Longrightarrow(\mathbf{i i})]$ From (i), (5.17) and (5.18), for all $\mathbf{h} \in C_{c}^{\infty}\left([0, T], \mathbb{R}^{n}\right)$, we have

$$
\begin{aligned}
0 & =\int_{[0, T]}\left(\mathfrak{M}(t) \cdot \mathbf{h}(t)+\mathfrak{N}(t) \cdot \mathbf{h}^{\prime}(t)\right) d \mathfrak{m}_{1}(t) \\
& =\int_{[0, T]}\left(\mathfrak{P}^{\prime}(t) \cdot \mathbf{h}(t)+\mathfrak{N}(t) \cdot \mathbf{h}^{\prime}(t)\right) d \mathfrak{m}_{1}(t) \\
& =\int_{[0, T]}\left(-\mathfrak{P}(t) \cdot \mathbf{h}^{\prime}(t)+\mathfrak{N}(t) \cdot \mathbf{h}^{\prime}(t)\right) d \mathfrak{m}_{1}(t) \\
& =\int_{[0, T]}(\mathfrak{N}(t)-\mathfrak{P}(t)) \cdot \mathbf{h}^{\prime}(t) d \mathfrak{m}_{1}(t) .
\end{aligned}
$$

Hence using the DuBois-Reymond lemma (6], Lemma 1.8, p.15) we obtain that there exists $c \in \mathbb{R}^{n *}$ s.t. $\mathfrak{N}(t)=\mathfrak{P}(t)+c \quad \mathfrak{m}_{1}$-a.e. $t \in[0, T]$ which is (ii).

$[(\mathbf{i i}) \Longrightarrow(\mathbf{i})]$ For all $\mathbf{h} \in C_{0,0}^{1}\left([0, T], \mathbb{R}^{n}\right)$, note that $\int_{[0, T]} c \cdot \mathbf{h}^{\prime}(t) d \mathfrak{m}_{1}(t)=\int_{0, T]}(c$. $\mathbf{h})^{\prime}(t) d \mathfrak{m}_{1}(t)=c \cdot \mathbf{h}(T)-c \cdot \mathbf{h}(0)=0$. Using (ii) and (5.17), we have $D_{H, 1} \Phi(\mathbf{x}(\pi), \pi)$. $\mathbf{h}=\int_{[0, T]}\left(\mathfrak{M}(t) \cdot \mathbf{h}(t)+\mathfrak{N}(t) \cdot \mathbf{h}^{\prime}(t)\right) d \mathfrak{m}_{1}(t)=\int_{[0, T]}\left(\mathfrak{P}^{\prime}(t) \cdot \mathbf{h}(t)+\mathfrak{P}(t) \cdot \mathbf{h}^{\prime}(t)\right) d \mathfrak{m}_{1}(t)+$ $\int_{[0, T]} c \cdot \mathbf{h}^{\prime}(t) d \mathfrak{m}_{1}(t)$, and using (5.18), we obtain $D_{H, 1} \Phi(x(\pi), \pi) \cdot \mathbf{h}=0$ which is (i).

Lemma 5.9. Under (BSOL1), (BINT1), (BINT2) and (BINT3), the condition (BCON) is equivalent to the linear independence of $D_{H, 1} G_{1}\left(x\left(\pi_{0}\right), \pi_{0}\right), \ldots, D_{H, 1} G_{k}\left(x\left(\pi_{0}\right), \pi_{0}\right)$, $D_{H, 1} H_{1}\left(x\left(\pi_{0}\right), \pi_{0}\right), \ldots, D_{H, 1} H_{\ell}\left(x\left(\pi_{0}\right), \pi_{0}\right)$ on $C_{0,0}^{1}\left([0, T], \mathbb{R}^{n}\right)$.

Proof. We want to use Lemma 5.8 with $\phi=\psi_{\lambda, \mu}$, where $\psi_{\lambda, \mu}$ is defined in (BCON). About the assumptions (i-iv) of Lemma 5.5, note that (i) is a consequence of (BINT2), (ii) is a consequence of (BSOL1), (iii) is a consequence of (BINT1), (iv) is a consequence of (BINT2). Hence from Lemma [5.5. we know that $\Phi: C^{1}([0, T], M) \times$ $P \rightarrow \mathbb{R}$, defined by $\Phi(\mathbf{x}, \pi):=\int_{0}^{T} \phi\left(t, \mathbf{x}(t), \mathbf{x}^{\prime}(t), \pi\right) d t=\int_{0}^{T} \psi_{\lambda, \mu}\left(t, \mathbf{x}(t), \mathbf{x}^{\prime}(t), \pi\right) d t$, is Hadamard differentiable at $x\left(\pi_{0}\right)$. To realize a proof, we proceeed doing a double contraposition. The negation of (B6) is equivalent to: $\exists(\lambda, \mu) \in \mathbb{R}^{k} \times \mathbb{R}^{\ell} \backslash\{(0,0)\}$, $\exists c_{\lambda, \mu} \in \mathbb{R}^{n *}$ s.t. 
$D_{H, 3} \psi_{\lambda, \mu}\left(t, x\left(\pi_{0}\right)(t), x\left(\pi_{0}\right)^{\prime}(t), \pi_{0}\right)=$

$\int_{[0, t]} D_{H, 2} \psi_{\lambda, \mu}\left(s, x\left(\pi_{0}\right)(s), x\left(\pi_{0}\right)^{\prime}(s), \pi_{0}\right) d \mathfrak{m}_{1}(s)+c_{\lambda, \mu} \quad \mathfrak{m}_{1}$-a.e. $t \in[0, T] . \quad$ Using

Lemma 5.8, this last assertion is equivalent to: $\exists(\lambda, \mu) \in \mathbb{R}^{k} \times \mathbb{R}^{\ell} \backslash\{(0,0)\}$ s.t. $D_{H, 1} \Phi\left(x\left(\pi_{0}\right), \pi_{0}\right)=0$ on $C_{0,0}^{1}\left([0, T], \mathbb{R}^{n}\right)$, i.e.

$\sum_{1 \leq i \leq k} \lambda_{i} D_{H, 1} G_{i}\left(x\left(\pi_{0}\right), \pi_{0}\right)+\sum_{1 \leq j \leq \ell} \mu_{j} D_{H, 1} H_{j}\left(x\left(\pi_{0}\right), \pi_{0}\right)=0$ on $C_{0,0}^{1}\left([0, T], \mathbb{R}^{n}\right)$. This last assertion means the linear dependence of $D_{H, 1} G_{1}\left(x\left(\pi_{0}\right), \pi_{0}\right), \ldots$, $D_{H, 1} H_{\ell}\left(x\left(\pi_{0}\right), \pi_{0}\right)$ on $C_{0,0}^{1}\left([0, T], \mathbb{R}^{n}\right)$.

\subsection{The dual space of $C_{0,0}^{1}\left([0, T], \mathbb{R}^{n}\right)$.}

Lemma 5.10. Let $V$ and $W$ be two real normed spaces. When $\left(v^{*}, w^{*}\right) \in V^{*} \times$ $W^{*}$, we consider the direct sum of $v^{*}$ and $w^{*}, v^{*} \oplus w^{*} \in(V \times W)^{*}$, defined by $v^{*} \oplus w^{*}(v, w):=v^{*}(v)+w^{*}(w)$ for all $(v, w) \in V \times W$. We define the operator $\mathcal{S}: V^{*} \times W^{*} \rightarrow(V \times W)^{*}$ by setting $\mathcal{S}\left(v^{*}, w^{*}\right):=v^{*} \oplus w^{*}$ when $\left(v^{*}, w^{*}\right) \in V^{*} \times W^{*}$. Then $\mathcal{S}$ is a topological isomorphism from $V^{*} \times W^{*}$ onto $(V \times W)^{*}$.

This result is given in [1] (Lemme 2, p.114) where its proof is left as an exercice since it is very easy. We consider $A f\left([0, T], \mathbb{R}^{n}\right)$, the set of the restrictions to $[0, T]$ of the affine functions from $\mathbb{R}$ into $\mathbb{R}^{n}$. Note that $\alpha \in A f\left([0, T], \mathbb{R}^{n}\right)$ means that there exists $(\eta, \sigma) \in \mathbb{R}^{n} \times \mathbb{R}^{n}$ s.t. $\alpha(t)=t \eta+\sigma$ for all $t \in[0, T]$. Clearly $A f\left([0, T], \mathbb{R}^{n}\right)$ is a vector subspace of $C^{1}\left([0, T], \mathbb{R}^{n}\right)$.

Lemma 5.11. $C^{1}\left([0, T], \mathbb{R}^{n}\right)=A f\left([0, T], \mathbb{R}^{n}\right) \oplus C_{0,0}^{1}\left([0, T], \mathbb{R}^{n}\right)$ (topological direct sum).

Proof. When $\varphi \in A f\left([0, T], \mathbb{R}^{n}\right) \cap C_{0,0}^{1}\left([0, T], \mathbb{R}^{n}\right)$, we have $\varphi(t)=t \eta+\sigma$ for all $t \in[0, T]$. Since $\varphi \in C_{0,0}^{1}\left([0, T], \mathbb{R}^{n}\right)$ we have $0=\varphi(0)=\sigma$ and $0=\varphi(T)=T \eta+\sigma$ which imply $\sigma=\eta=0$, and consequently $\varphi=0$. Hence we have established the following property on the algebraic direct sum : $A f\left([0, T], \mathbb{R}^{n}\right) \oplus^{a} C_{0,0}^{1}\left([0, T], \mathbb{R}^{n}\right) \subset$ $C^{1}\left([0, T], \mathbb{R}^{n}\right)$. When $\varphi \in C^{1}\left([0, T], \mathbb{R}^{n}\right)$, we set $\eta:=-\frac{1}{T}(\varphi(0)-\varphi(T)), \sigma:=\varphi(0)$ and $\alpha(t):=t \eta+\sigma$ for all $t \in[0, T]$. Hence we have $\alpha \in A f\left([0, T], \mathbb{R}^{n}\right)$. We define $\psi:[0, T] \rightarrow \mathbb{R}^{n}$ by setting $\psi(t):=\varphi(t)+\frac{t}{T}(\varphi(0)-\varphi(T))-\varphi(0)$ for all $t \in[0, T]$. Note that $\psi \in C^{1}\left([0, T], \mathbb{R}^{n}\right), \psi(0)=\varphi(0)-\varphi(0)=0$, and $\psi(T)=\varphi(T)+(\varphi(0)-$ $\varphi(T))-\varphi(0)=0$. Hence we have $\psi \in C_{0,0}^{1}\left([0, T], \mathbb{R}^{n}\right)$. We see that $\alpha(t)+\psi(t)=\varphi(t)$ for all $t \in[0, T]$, i.e. $\alpha+\psi=\varphi$. And so we have proven that $A f\left([0, T], \mathbb{R}^{n}\right) \oplus^{a}$ $C_{0,0}^{1}\left([0, T], \mathbb{R}^{n}\right)=C^{1}\left([0, T], \mathbb{R}^{n}\right)$. Since $\operatorname{dim} A f\left([0, T], \mathbb{R}^{n}\right)<+\infty$ the subspace $A f\left([0, T], \mathbb{R}^{n}\right)$ is complete, since $C_{0,0}^{1}\left([0, T], \mathbb{R}^{n}\right)$ is closed in $C^{1}\left([0, T], \mathbb{R}^{n}\right)$ which is complete, when obtain the announced conclusion (cf. [19, Corollary 1.5, p.388)

From this lemma, we can write

$$
C^{1}\left([0, T], \mathbb{R}^{n}\right)=A f\left([0, T], \mathbb{R}^{n}\right) \times C_{0,0}^{1}\left([0, T], \mathbb{R}^{n}\right) .
$$

Using Lemma 5.10 for $V=A f\left([0, T], \mathbb{R}^{n}\right)$ and $W=C_{0,0}^{1}\left([0, T], \mathbb{R}^{n}\right)$, and denoting $\mathcal{S}_{1}:\left(A f\left([0, T], \mathbb{R}^{n}\right)\right)^{*} \times\left(C_{0,0}^{1}\left([0, T], \mathbb{R}^{n}\right)\right)^{*} \rightarrow\left(C^{1}\left([0, T], \mathbb{R}^{n}\right)\right)^{*}$, defined by $\mathcal{S}_{1}(\chi, \Lambda):=\chi \oplus \Lambda$, we obtain

$$
\mathcal{S}_{1} \in \operatorname{Isom}\left(\left(A f\left([0, T], \mathbb{R}^{n}\right)\right)^{*} \times\left(C_{0,0}^{1}\left([0, T], \mathbb{R}^{n}\right)\right)^{*},\left(C^{1}\left([0, T], \mathbb{R}^{n}\right)\right)^{*}\right) .
$$

Using Lemma 5.10 for $V=\mathbb{R}^{n}$ and $W=C^{0}\left([0, T], \mathbb{R}^{n}\right)$, and denoting $\mathcal{S}_{2}:\left(\mathbb{R}^{n}\right)^{*} \times$ $\left(C^{0}\left([0, T], \mathbb{R}^{n}\right)\right)^{*} \rightarrow\left(\mathbb{R}^{n} \times C^{0}\left([0, T], \mathbb{R}^{n}\right)\right)^{*}$, defined by $\mathcal{S}_{2}(\beta, \Theta):=\beta \oplus \Theta$, we obtain

$$
\mathcal{S}_{2} \in \operatorname{Isom}\left(\left(\mathbb{R}^{n}\right)^{*} \times\left(C^{0}\left([0, T], \mathbb{R}^{n}\right)\right)^{*},\left(\mathbb{R}^{n} \times C^{0}\left([0, T], \mathbb{R}^{n}\right)\right)^{*}\right) .
$$


The operator $\mathcal{T}: C^{1}\left([0, T], \mathbb{R}^{n}\right) \rightarrow \mathbb{R}^{n} \times C^{0}\left([0, T], \mathbb{R}^{n}\right)$, defined by $\mathcal{T}(x):=\left(x(0), x^{\prime}\right)$, is a topological isomorphism. Hence (cf. [12], Theorem 4.13.4, p.173), its adjoint satisfies the following property.

$$
\mathcal{T}^{*} \in \operatorname{Isom}\left(\left(\mathbb{R}^{n} \times C^{0}\left([0, T], \mathbb{R}^{n}\right)^{*},\left(C^{1}\left([0, T], \mathbb{R}^{n}\right)\right)^{*}\right) .\right.
$$

We denote by $\mathcal{R}_{F}:\left(\mathbb{R}^{n}\right)^{*} \rightarrow \mathbb{R}^{n}$ the isomorphism of F. Riesz and Fréchet ([23], p. 81$)$, and by $\mathcal{R}_{M}:\left(C^{0}\left([0, T], \mathbb{R}^{n}\right)\right)^{*} \rightarrow N B V\left([0, T], \mathbb{R}^{n}\right)$ the topological isomorphism of F. Riesz and Markov ([16], p. 365). We define the operator $\left(\mathcal{R}_{F}, \mathcal{R}_{M}\right)$ : $\left(\mathbb{R}^{n}\right)^{*} \times\left(C^{0}\left([0, T], \mathbb{R}^{n}\right)\right)^{*} \rightarrow \mathbb{R}^{n} \times N B V\left([0, T], \mathbb{R}^{n}\right)$, defined by $\left(\mathcal{R}_{F}, \mathcal{R}_{M}\right)(\beta, \Theta):=$ $\left(\mathcal{R}_{F}(\beta), \mathcal{R}_{M}(\Theta)\right)$. We easily verify the following property.

$$
\left(\mathcal{R}_{F}, \mathcal{R}_{M}\right) \in \operatorname{Isom}\left(\left(\mathbb{R}^{n}\right)^{*} \times\left(C^{0}\left([0, T], \mathbb{R}^{n}\right)\right)^{*}, \mathbb{R}^{n} \times N B V\left([0, T], \mathbb{R}^{n}\right)\right) .
$$

Now we can establish the following result.

Lemma 5.12. There exists an inner product on $\left(C_{0,0}^{1}\left([0, T], \mathbb{R}^{n}\right)^{*} \times\left(C_{0,0}^{1}\left([0, T], \mathbb{R}^{n}\right)^{*}\right.\right.$ which is continuous with respect to the usual topology of the product space.

Proof. We consider the operator

$$
\text { in }:\left(C_{0,0}^{1}\left([0, T], \mathbb{R}^{n}\right)^{*} \rightarrow\left(A f\left([0, T], \mathbb{R}^{n}\right)\right)^{*} \times\left(C_{0,0}^{1}\left([0, T], \mathbb{R}^{n}\right)^{*}, \text { in }(\Lambda):=(0, \Lambda) .\right.\right.
$$

This operator is linear continuous and injective. We introduce the following operator:

$$
\Gamma:=\left(\mathcal{R}_{F}, \mathcal{R}_{M}\right) \circ \mathcal{S}_{2}^{-1} \circ\left(\mathcal{T}^{*}\right)^{-1} \circ \mathcal{S}_{1} \circ \text { in }
$$

From (5.20), (5.21), (5.22), (5.23) and (5.24), we obtain that $\Gamma$ is linear continuous and injective. Now we build the operator $\Delta$ from $\left(C_{0,0}^{1}\left([0, T], \mathbb{R}^{n}\right)^{*} \times\right.$ $\left(C_{0,0}^{1}\left([0, T], \mathbb{R}^{n}\right)^{*}\right.$ into $\left(\mathbb{R}^{n} \times N B V\left([0, T], \mathbb{R}^{n}\right) \times\left(\mathbb{R}^{n} \times N B V\left([0, T], \mathbb{R}^{n}\right)\right)\right.$ by setting

$$
\Delta:=\left(\Gamma \circ p r_{1}, \Gamma \circ p r_{2}\right)
$$

where $p r_{1}$ and $p r_{2}$ are the projections of $\left(C_{0,0}^{1}\left([0, T], \mathbb{R}^{n}\right)^{*} \times\left(C_{0,0}^{1}\left([0, T], \mathbb{R}^{n}\right)^{*}\right.\right.$. From (5.25), we obtain that $\Delta$ is continuous on $\left(C_{0,0}^{1}\left([0, T], \mathbb{R}^{n}\right)^{*} \times\left(C_{0,0}^{1}\left([0, T], \mathbb{R}^{n}\right)^{*}\right.\right.$.

We consider the inner product $(\cdot \mid \cdot)_{0}$ on $\mathbb{R}^{n} \times N B V\left([0, T], \mathbb{R}^{n}\right)$ defined by

$$
\left(\left(\xi_{1}, g_{1}\right) \mid\left(\xi_{2}, g_{2}\right)\right)_{0}:=\left(\xi_{1} \mid \xi_{2}\right)_{\mathbb{R}^{n}}+\int_{0}^{T}\left(g_{1}(t) \mid g_{2}(t)\right)_{\mathbb{R}^{n}} d t+\left(g_{1}(T) \mid g_{2}(T)\right)_{\mathbb{R}^{n}}
$$

This inner product is continuous withe respect to the usual norm of

$\mathbb{R}^{n} \times N B V\left([0, T], \mathbb{R}^{n}\right)$. The functional $(\cdot \mid \cdot):=(\cdot \mid \cdot)_{0} \circ \Delta$ is an inner product on $\left(C_{0,0}^{1}\left([0, T], \mathbb{R}^{n}\right)^{*} \times\left(C_{0,0}^{1}\left([0, T], \mathbb{R}^{n}\right)^{*}\right.\right.$ which is continuous as a composition of continuous mappings.

5.5. Proof of Theorem 5.1. Conclusion (I). Our strategy is to use Corollary 3.2. We start by doing the dictionary between the notation of Corollary 3.2 and the notation of Theorem 5.1, Let $\mathbb{X}:=C^{1}\left([0, T], \mathbb{R}^{n}\right), \mathbb{A}:=C_{a_{0}, a_{T}}^{1}\left([0, T], \mathbb{R}^{n}\right), \mathbb{S}:=$ $C_{0,0}^{1}\left([0, T], \mathbb{R}^{n}\right), \mathbb{G}:=C^{1}([0, T], M), f^{0}(x, \pi)=J(x, \pi)=\int_{0}^{T} L\left(t, x(t), x^{\prime}(t), \pi\right) d t$, $g_{i}^{0}(x, \pi)=G_{i}(x, \pi)=\int_{0}^{T} \mathfrak{g}_{i}\left(t, x(t), x^{\prime}(t), \pi\right) d t$ when $1 \leq i \leq k, h_{j}^{0}(x, \pi)=H_{j}(x, \pi)=$ $\int_{0}^{T} \mathfrak{h}_{j}\left(t, x(t), x^{\prime}(t), \pi\right) d t$ when $1 \leq j \leq \ell$, and $\underline{z}(\pi)=x(\pi)$.

Now we consider the assumptions of Corollary 3.2. ( $\left.A^{0} \mathrm{DUA}\right)$ is fulfilled by using Lemma 5.12. ( $\left.A^{0} \mathrm{SOL} 1\right)$ is a consequence of (BSOL1), and $\left(A^{0} \mathrm{SOL} 2\right)$ is a consequence of (BSOL2).

To show that $\left(A^{0} \mathrm{FON} 1\right)$ is fulfilled, we want to use Lemma 5.5. hence we ought 
to prove that the assumptions of Lemma [5.5 are fulfilled. In the proof of Lemma 5.9, we have noted that (Bsol1), (BINT1), (Bint2) and (BINT3) ensure that the conditions (i-v) of Lemma 5.5 are fulfilled and consequently the conclusions (a), (b) and (c) of Lemma 5.5 hold. Hence $\left(A^{0}\right.$ FON1) results from the conclusions (b) and (c) of Lemma 5.5. ( $\left.A^{0} \mathrm{CON} 1\right)$ results from the conclusion (a) of lemma 5.5. $\left(A^{0} \mathrm{CON} 2\right)$ results from $(\mathrm{BCON})$ and of Lemma 5.8. Then we can use the conclusion $(\alpha)$ of Corollary 3.2 which permits to ensure that the conclusion (I) of Theorem 5.1 is proven.

Conclusion (II). In the proof of the conclusion (I), we have yet proven that $\left(A^{0} \mathrm{SOL} 1\right),\left(A^{0} \mathrm{SOL} 2\right),\left(A^{0} \mathrm{FON} 1\right),\left(A^{0} \mathrm{CON} 1\right)$ and $\left(A^{0} \mathrm{CON} 2\right)$ are fulfilled. Replacing (Bsol2) by (Bsol2-bis), ( $A^{0}$ SOL2-bis) is fulfilled, and we can use the conclusion $(\beta)$ of Corollary 3.2 to obtain the conclusion (II) of Theorem 5.1 .

Conclusion (III). After the proofs of the previous conclusions, we know that the assumptions (i), (ii), (iii), (iv) and (v) of Lemma 5.5 are fulfilled. Note that (BINT3bis) implies that assumption (vi) of Lemma 5.5 holds. Also note that (BINT4) implies that the assumption (vii) of Lemma 5.5 holds. Hence we can use the conclusion (d) of Lemma 5.5. From this conclusion (d) we deduce that $\left(A^{0}\right.$ FON2) holds. Note that (Bsol2-ter) implies ( $A^{0}$ SOL2-ter). Hence we can use the conclusion $(\gamma)$ of Corollary 3.2 to obtain the conclusion (III) of Theorem 5.1.

\section{REFERENCES}

[1] V. Alexeev, V. Tihomirov, and S. Fomin, Commande optimale, french edition, MIR, Moscow, 1982.

[2] J. Blot, On the multiplier rules, Optimization 65(2) (2016) 947-955.

[3] J. Blot, and M.I. Koné, Euler-Lagrange equations for a delay variational problem, Nonauto. Dyn. Syst. 4 (2017) 52-61.

[4] J. F. Bonnans, and A. Shapiro, Optimization problems with perturbations: a guided tour, SIAM Rev. 40(2) (1998) 228-264.

[5] K.C. Border, Classical envelope theory, Caltech, Division of the Humanities and Social Sciences (2006) v.2015119:1420.

[6] G. Buttazzo, M. Giaquinta, and S. Hildebrandt, One-dimensional variational problems, Oxford University Press, Inc., New York, 1998.

[7] M. R. Caputo, Comparative dynamics via envelope methods in variational calculus, Rev. Econom. Stud., 57(4) (1990) 689-697.

[8] M. Carter, Foundations of mathematical economics, M.I.T. Press, Cambridge (Mass.), 2001.

[9] J. Dieudonné, Éléments d'analyse, tome 1: fondements de l'analyse moderne, french edition, Gauthier-Villars, Paris 1969.

[10] T.M. Flett, Differential analysis, Cambridge University Press, Cambridge, N.Y., 1980.

[11] C. Fourgeaud, and A. Perrot, Calcul économique et microéconomie appronfondie, Economica, Paris, 1990.

[12] A. Friedman, Foundations of modern analysis, Dover Publ., Inc., New York, N.Y., 1982.

[13] H. Helson, Harmonic analysis, Wadsworth, Inc., Belmont, California, 1991.

[14] A.D. Ioffe, and V.M. Tihomirov, Theory of extremal problems, North Holland Publ. Comp., Amsterdam, 1979.

[15] L.V. Kantorovitch, and G.P. Akilov, Analyse fonctionnelle; tome 1: opérateurs et fonctionnelles linéaires, french edition, MIR, Moscow, 1981.

[16] A.N. Kolmogorov, and S.V. Fomin, Éléments de la théorie des fonctions et de l'analyse fonctionnelle, french edition, MIR, Moscow, 1974.

[17] J.T. LaFrance, and L. Dwayne Barney, The envelope theorem in dynamic optimization, J. Econom. Dynam. Control, 15 (1991) 355-383.

[18] S. Lang, Algebra, Addison-Wesley Publ. Comp., Reading (Mass.), 1965.

[19] S. Lang, Real and functional analysis, third edition, Springer-Verlag New York, Inc., 1993.

[20] D. Léonard, and N.V. Long, Optimal control theory and static optimization in economics, Cambridge University Press, Cambridge (Mass.), 1992. 
[21] P. Milgrom, and I. Segal, Envelope theorems for arbitrary choice sets, Econometrica, 70(2) (2002) 581-601.

[22] O. Morand, K. Reffet, and S. Tarafdar, Generalized envelope theorems: applications to dynamic programming, J. Optim. Theory Appl. (2018) https://doi.org/10.1007:s-10957-0181241-5.

[23] W. Rudin, Real and complex analysis, third edition, McGraw-Hill Book Company, New York, 1986.

[24] E. Silverberg, The Le Chapelier principle as a corollary to a generalized envelope theorem, J. Econom. Theory 3 (1971) 146-155.

[25] E. Silverberg, A revision of comparative statics methodology in Economics or, How to do comparative statics on the back of an envelope, J. Econom. Theory 7 (1974) 159-172.

[26] H. Yilmaz, A generalization of multiplier rules for infinite-dimensional optimization problems, Optimization 70(8) (2021) 1825-1835.

JoËL Blot: Laboratoire SAMM EA4543,

Université Paris 1 Panthéon-Sorbonne, centre P.M.F.,

90 Rue de Tolbiac, 75634 Paris Cedex 13, France.

Email address: blot@univ-paris1.fr

Hasan Yilmaz: Laboratoire LPSM, UMR 8001, Université de Paris, bâtiment Sophie Germain, 8 place Aurélie Nemours, 75013 Paris, France.

Email address: yilmaz.research@gmail.com 